\title{
Elterliches schulbezogenes Unterstützungshandeln
}

I: Und dann haben Sie noch das Unterstützen erwähnt. Was muss ich mir darunter vorstellen?

H11: Ja, verschiedene Arten von Unterstützung. Emotionell, auch, auch-. Wenn sie in Mathematik Probleme hat, dass wir etwas lösen können. Oder-. Ja, jemanden anrufen-. Verschiedene Unterstützungen. Für sie da zu sein. Immer. Ja. [...] Unterstützen, dass sie merkt, dass sie nicht alleine ist, wenn sie ein Problem hat. Das heißt auch im Leben allgemein, nicht nur in Kleinigkeiten oder-. Ja. (...) Das ist beim Kind auch wichtig oder. Dass es weiß, dass es irgendwo hingehört. Und dass, ja-. Jemand es gerne hat, es liebhat, nicht wahr. (Interview G2, 00:11:18)

Der Ausdruck «Elterliche schulbezogene Unterstützung» wurde für die vorliegende Studie in Anlehnung an den Terminus «Stützsysteme» gewählt, der sich in sog. Angebots-Nutzungsmodellen in der Schul- und Unterrichtsforschung (vgl. Fend, 2006, 2008; Helmke, 2017; Reusser \& Pauli, 2010) findet und da zusätzliche Ressourcen bezeichnet, die den Akteuren auf den verschiedenen Ebenen des Bildungssystems auf der Angebots- und auf der Nutzungsseite zur Verfügung stehen. So können Lehrkräfte zur Optimierung ihres «Angebotshandelns» beispielsweise auf diverse didaktische, schulpsychologische, sonder- und sozialpädagogische Stützangebote zurückgreifen und den Schülerinnen und Schülern stehen insbesondere ihre Familienmitglieder, aber auch Peers und schulinterne und -externe Nachhilfeangebote stützend zur Optimierung der «Nutzungsqualität»(Fend, 2008, S. 22) zur Seite. Damit wird deutlich, dass «Unterstützen» ein Tun bezeichnet, das auf ein anderes Tun bezogen ist, welches eigentlich im Fokus steht. Laut Duden besitzt der Ausdruck zwei Bedeutungskerne: a) jemandem materiell oder «mit Rat und Tat» «bei etwas behilflich sein» und b) «sich für 
jemandes Angelegenheiten o. Ä. einsetzen und dazu beitragen, dass jemand/etwas Fortschritte macht, Erfolg hat».

«Unterstützen» impliziert mit anderen Worten einen Aktor und einen Koaktor (vgl. Aebli, 1993, S. 122-127): den Unterstützer und den Unterstützten ${ }^{1}$. Letzterer befindet sich in einer Situation, die er nicht selbst bewältigen $\mathrm{kann}^{2}$, sondern in der er auf zusätzliche Ressourcen angewiesen ist, die ihm der Unterstützer zur Verfügung stellt, indem er a) gemäß dem ersten Bedeutungskern mit ihm direkt in Transaktion tritt - er seine materiellen und immateriellen Güter also direkt an den Bedürftigen übergibt - und/oder b) gemäß dem zweiten Bedeutungskern bei einem zweiten Koaktor zugunsten des Bedürftigen seinen Ressourceneinsatz leistet, sich für ihn bei diesem «einsetzt» und ihm so im besten Fall zu einem «Fortschritt» oder «Erfolg» (Duden) verhilft. Dabei wird deutlich, dass dieser Dritte in einer Machtposition ist, insofern als er einerseits den «Angelegenheiten» (Duden) des Unterstützten «Fortschritt» und «Erfolg» zuerkennen kann, andererseits dem Unterstützenden Gehör schenken kann, wenn dieser sich für den Bedürftigen einsetzt. Machtasymmetrie wird durch die erste Wortdefinition aber auch zwischen dem Unterstützenden und dem Unterstützten impliziert: Sofern der Unterstützte die zusätzlichen Ressourcen, die der Unterstützer kontrolliert, auch wirklich begehrt, von seiner Seite also ein Transaktionsinteresse besteht, liegt auch hier eine klassische Machtkonstellation vor (vgl. Esser, 2000c, S. 385-410).

Überträgt man diese Überlegungen auf die Situation, in der sich Eltern mit ihren Kindern im Kontext des Schulsystems befinden, so wird klar, dass hier beide Bedeutungskerne des Verbs «unterstützen» relevant sind: Eltern können a) mittels direkter Hilfestellungen, die sie zu Hause realisieren, versuchen, das Handeln ihres Kindes in Schule und Unterricht zu fördern und können b) ebenso den Versuch unternehmen, über die Kommunikation mit der Klassenlehrkraft und weiteren Akteuren des Schulsystems zugunsten des Kindes Einfluss zu nehmen. In welchem Ausmaß und in welcher Qualität sie das eine und/oder andere tun, dürfte davon abhängen,

\footnotetext{
${ }^{1}$ Wenn immer möglich werden in der vorliegenden Arbeit beide Geschlechtsformen oder aber eine geschlechtsneutrale Bezeichnung verwendet. In Einzelfällen, in denen die Verwendung geschlechtsneutraler oder beider Geschlechtsformen dem Lese- und Verstehensfluss zu abträglich ist (wie in der vorliegenden Passage) wird zwar auf die weibliche Form verzichtet, ist aber mitgemeint.

${ }^{2}$ Eine Dysfunktionalität liegt dann vor, wenn der Unterstützungsbedarf lediglich vom Aktor, nicht aber vom rezipierenden Koaktor wahrgenommen wird (vgl. Autonomieunterstützung vs. Strukturgebung vs. Zwang, Abschnitt 2.2.2.4).
} 
- ob sie überhaupt ein Problem mit Bezug auf die schulische Situation ihres Kindes wahrnehmen und somit einen Bedarf für einen Ressourceneinsatz ihrerseits erkennen,

- für wie bedeutsam sie dieses Problem halten,

- wie sie ihre eigenen Ressourcen zur erfolgreichen Bearbeitung des Problems unter den gegebenen situativen Bedingungen einschätzen und

- welche alternativen Ressourcen sie als zugänglich und allenfalls als nützlich erkennen.

$\mathrm{Ob}$ und wie das Kind oder die Lehrkraft die elterlichen Ressourcen überhaupt nutzen, dürfte von deren korrespondierenden Einschätzungen abhängen:

- Nehmen sie ihrerseits einen Bedarf wahr? Falls ja,

- welche Dringlichkeit einer Problemlösung erkennen sie?

- Wie schätzen sie ihre eigenen Ressourcen zur effektiven Bearbeitung des Problems ein und

- worin besteht für sie allenfalls der Mehrwert der elterlichen Ressourcen unter den gegebenen Bedingungen? - Soll darauf zugegriffen werden? Ist die Situation so, dass die Eltern einbezogen werden müssen? Gäbe es Alternativen?

Die Skizze möglicher Gedanken der unmittelbar Beteiligten verdeutlicht, dass elterliche schulbezogene Unterstützung als genuin soziales Handeln konzipiert werden muss, das von wechselseitigen Wahrnehmungs- und Entscheidungsprozessen sowie von institutionellen Bedingungen geprägt wird. Bevor die Situation noch detaillierter aus verschiedenen Blickwinkeln ausgelotet wird, soll im Folgenden Hartmut Essers «Allgemeine Handlungstheorie» (1999a) erläutert werden, die als soziologische Theorie einerseits die sozialen und institutionellen Bedingungen des Handelns genau in den Blick zu nehmen vermag und andererseits gesellschaftliche Phänomene (wie beispielsweise die sozialen Disparitäten bei der Bildungsbeteiligung und dem Bildungserfolg hierzulande) als aggregierter Effekt des Handelns von Akteuren konzipiert. Das Besondere besteht darin, dass es Essers Theorie nicht, wie die meisten anderen soziologischen Akteurstheorien, bei relativ unspezifischen Aussagen über bestimmte «Handlungsorientierungen» der Akteure bewenden lässt, sondern detailliert zu erklären sucht, wie es zur Handlungsselektion beim Individuum kommt. Den Erklärungsansatz hierfür findet er in der ursprünglich aus der Ökonomie stammenden Wert-Erwartungs-Theorie, die in der Soziologie seit den Arbeiten von Raymond Boudon (1974) zu Bildungsentscheidungen breit diskutiert wurde und von Esser auch mit Blick auf den Einfluss, 
den Erwartungs-Wert-Konzeptionen in den letzten sechzig Jahren in der Psychologie entfaltet haben (z. B. Atkinson, 1957; Eccles-Parsons et al., 1983; Lewin, 1926; Pekrun, 2006; Tolman, 1932), als zentralen Mechanismus der Handlungswahl in seine Theorie integriert wurde $^{3}$. Als soziologische Theorie unterlässt es seine Konzeption zwar, das Zusammenspiel zwischen Persönlichkeitsmerkmalen, individuellen Überzeugungen und den Entscheidungsprozessen genauer zu modellieren $^{4}$, dafür werden aber die Informationsverarbeitungsprozesse, die bei der Situationsanalyse und Handlungswahl ablaufen, in einem Maß detailliert erklärt, wie dies motivationspsychologische Erwartungs-Wert-Theorien (vgl. Kapitel 5) in der Regel nicht leisten. Ebenso wird bei Esser deutlich, wie sich «aufgeklärte» (Becker, R., 2017b, S. 111) sozialwissenschaftliche Rational Choice-Theorien, wie beispielsweise diejenige von Boudon $(1974,1980)$ (vgl. Abschnitt 3.1.1), von klassischen ökonomischen Rational Choice-Ansätzen unterscheiden.

Essers Handlungstheorie bietet sich wegen dieser integrierten Sicht auf individuelle und kollektiv-gesellschaftliche Prozesse sowie wegen ihrer Anschlussfähigkeit an sozialkognitive Motivations- und Entscheidungstheorien an dieser Stelle zur Darstellung des gemeinsamen Menschenbildes an, welches den verschiedenen soziologischen und psychologischen erwartungswert-theoretischen Konzeptionen zugrunde liegt, die im Verlauf der kommenden Kapitel noch eingehender erläutert werden ${ }^{5}$.

\subsection{Handlungstheoretische Grundlegung}

Der klassische Strukturfunktionalismus in der Tradition von Talcott Parsons (1991) konzipierte den Handelnden im Kern als Vollzieher sozialer Normen bzw. institutioneller Vorgaben, der mit seinem Handeln gleichzeitig die Funktion erfüllt, die gesellschaftliche Ordnung zu sichern. Der Schlüsselbegriff hierbei ist derjenige der sozialen Rolle. Mit jeder gesellschaftlichen Position sind soziale Normen, Erwartungen und Handlungsmuster verbunden, die das Individuum nach dem klassischen soziologischen Menschenbilde des homo sociologicus (Dahrendorf, 2006) im Prozess der Sozialisation entweder freiwillig in sein Wertesystem

\footnotetext{
${ }^{3}$ Esser sieht sich denn auch mitunter mit dem Vorwurf der «Psychologisierung der Soziologie» konfrontiert (z. B. Kron, 2004).

${ }^{4} \mathrm{Zu}$ weiteren Schwächen soziologischer Erwartungs-Wert-Theorien aus psychologischer Perspektive vgl. Maaz et al. (2006, S. 315).

${ }^{5}$ Vgl. auch Fends Würdigung der Theorie Essers und des Beitrags, den dessen Handlungsmodell zur Entwicklung einer «Neuen Theorie der Schule» geleistet habe (Fend, 2006, S. 151-152).
} 
übernimmt, weil sie von ihm als bewährt anerkannt werden, oder aber sich nur notgedrungen vordergründig $\mathrm{zu}$ eigen macht und befolgt, weil es bei Abweichungen Sanktionen befürchtet oder bei konformem Verhalten Belohnungen erwartet:

Soziale Rollen sind ein Zwang, der auf den Einzelnen ausgeübt wird - mag dieser als eine Fessel seiner privaten Wünsche oder als ein Halt, der ihm Sicherheit gibt, erlebt werden. Dieser Charakter von Rollenerwartungen beruht darauf, dass die Gesellschaft Sanktionen zur Verfügung hat, mit deren Hilfe sie die Vorschriften zu erzwingen vermag. Wer seine Rolle nicht spielt, wird bestraft; wer sie spielt, wird belohnt, zumindest aber nicht bestraft. (Dahrendorf, 2006, S. 40-41)

Der Zwang, von dem Dahrendorf spricht, ist Ausdruck eines Systems von Werten, das in einer geteilten symbolischen Ordnung, einer Kultur, konsensuell existiert und in Form von wechselseitigen Erwartungen strukturierend auf die Handlungsentscheidungen des Einzelnen einwirkt. Handeln heißt für Parsons (1991) vor diesem Hintergrund, sich bei der Auswahl der Ziele und der Mittel zur Erreichung der eigenen Interessen immer bewusst zu machen, was von einem erwartet wird, und sich an den gesellschaftlich-kulturell gebotenen Handlungsoptionen $\mathrm{zu}$ orientieren: «This fundamental relationship between need-dispositions of the personality, role-expectations of the social system and internalized-institutionalized [sic] value-patterns of the culture is the fundamental nodal point of the organization of systems of action» (Parsons, 1991, S. 363).

Gegen eine solche Sichtweise, in welcher der Mensch als «als passive Marionette undurchsichtiger normativer und struktureller Kräfte» (Giddens, 1988, S. 287) erscheint, und der wie Esser (1999a, S. 236) meint, insbesondere eine «explizite und präzise Selektionsregel für das Handeln» fehle, wenden sich die Vertreter der Theorie des Rational Choice. Sie postulieren, dass a) soziale Phänomene von den Handlungen der Individuen her erklärt werden müssten und b) diese Handlungen ihrerseits auf subjektiven rationalen Handlungsentscheidungen basierten (vgl. Diefenbach, 2009, S. 239). Orientiert an der Moralphilosophie David Humes $(1978,2006)$ wird rationales Handeln als zweckgerichtetes, intentionales Tun des Menschen konzipiert, das seinen eigenen Interessen, Zielen und Bedürfnissen dient, wobei Rationalität auf die Selektion zwischen Handlungsalternativen bezogen ist, auf den Prozess also, in dem wahrgenommene Handlungsoptionen nach deren Nutzen und Kosten beurteilt werden (Nutzenorientierung) und diejenige ausgewählt wird, die den höchsten Nutzen und die geringsten Kosten erwarten lässt (Maximierungsorientierung) (vgl. Jäger \& Meyer, 2007, S. 107). 
Die Handlungsalternativen werden mit anderen Worten von deren Konsequenzen her beurteilt. Von einem Nutzen wird dann gesprochen, wenn sich der Nutzenwert der Konsequenz im positiven Bereich befindet, von Kosten, wenn der Nutzenwert einer Handlungskonsequenz negativ ausfällt. In die Entscheidung für oder gegen eine bestimmte Handlungsoption fließt nebst der Einschätzung ihres Werts zur Erreichung eines Ziels auch ein, mit welcher Wahrscheinlichkeit dieses Ziel mit ihr erreicht werden kann. Entlang der beiden Grundmaximen: «Versuche dich vorzugsweise an solchen Handlungen, deren Folgen nicht nur wahrscheinlich, sondern Dir gleichzeitig auch etwas wert sind! Und meide ein Handeln, das schädlich bzw. zu aufwendig für Dich ist und/oder für Dein Wohlbefinden keine Wirkung hat!» (Esser, 1999b, S. 284, Hervorhebungen im Original), wird die Person aufgrund anthropologischer und evolutionärer Voraussetzungen (vgl. Esser, 1999a, S. 219-229) schließlich diejenige Handlung auswählen, die - soweit für sie ersichtlich - mit der höchsten Wahrscheinlichkeit zu derjenigen Konsequenz führt, die für sie subjektiv den höchsten Wert aufweist (vgl. Diefenbach, 2009, S. 245). Dieser Erwartungs-Wert-Zusammenhang ist der handlungstheoretische Kern aller Rational Choice-Ansätze und fungiert in der Soziologie als Subjective Expected Utility Theory (SEU) und in der Psychologie als Expectancy-Value Theory ${ }^{6}$.

Handeln als zielgerichtetes Tun bedeutet in der Terminologie von James S. Coleman (1990, S. 27-29), einem der wichtigsten Exponenten der Theorie der rationalen Wahl, immer der Einsatz von Ressourcen zur Produktion von solchen Ressourcen, die einem subjektiv als wichtig oder interessant erscheinen, aber von einem (noch) nicht kontrolliert werden. Wie Esser daran anschließend festhält, können Ressourcen «alle möglichen materiellen und immateriellen Dinge, aber auch gewisse Ereignisse, Zustände, Eigenschaften und Leistungen sein, an denen ein Akteur Interesse finden und die er unter Kontrolle haben oder bekommen kann» (Esser, 1999b, S. 38). Interesse und Kontrolle sind die beiden Größen, welche die Beziehung zwischen dem Akteur und den Ressourcen ausmachen:

Das Interesse leitet sich aus den grundlegenden Bedürfnissen und aus den Präferenzen, den Bewertungen also, ab: aus dem allgemeinen Bedürfnis nach physischem Wohlergehen und dem nach sozialer Anerkennung. [...] Die Kontrolle bezeichnet den Grad

\footnotetext{
${ }^{6}$ Im deutschen Sprachraum haben sich je nach wissenschaftlicher Disziplin die beiden Bezeichnungen «Wert-Erwartungstheorie» (Soziologie) und «Erwartungs-Wert-Theorie» (Psychologie) eingebürgert (vgl. Maaz et al., 2006). In der vorliegenden Studie wird aus Gründen der größeren Gewichtung psychologischer Ansätze fortan einheitlich der Terminus «Erwartungs-Wert-Theorie» bzw. «Erwartungs-Wert-Modell» verwendet.
} 
der Verfügbarkeit einer Ressource für den Einsatz im Handeln. Dieser Grad der Verfügbarkeit spiegelt sich in den subjektiven Erwartungen, die betreffende Ressource für eine bestimmte Handlung auch einsetzen zu können. Etwas technischer ausgedrückt: Die Ressourcen, die ein Akteur kontrolliert, sind der feasible set der Opportunitäten, die ihm in einer Situation zur Verfügung stehen. Sie sind das Kapital, das Budget, mit dem ein Akteur rechnen kann. (Esser, 1999a, S. 342, Hervorhebungen im Original)

Nun ist es allerdings so, dass viele Ziele nur mit Hilfe von anderen Akteuren erreichbar sind und die Kontrolle über Ressourcen folglich nur erlangt werden kann, wenn das Prinzip der Kooperation zum Tragen kommt. Esser sieht hierin im Einklang mit Coleman das grundlegende Moment der Sozietät des Menschen: Dem «Auseinanderfallen von Kontrolle über Ressourcen und von Interesse an Ressourcen» (Esser, 1999a, S. 342). Interdependenz zwischen den Akteuren ist die Folge dieses Umstandes: Akteur A kontrolliert Ressourcen, an denen Akteur $\mathrm{B}$ interessiert ist und umgekehrt ${ }^{7}$. Dabei ist es so, dass Interdependenzen kaum je ganz symmetrisch sind. Macht kommt dann ins Spiel, wenn ein Akteur über mehr Ressourcen die Kontrolle ausübt, die den anderen interessieren. Mit jeder Änderung bei Kontrolle und Interesse verändert sich die Machtkonstellation zwischen den Akteuren: «Relativ mächtiger ist ... nicht nur derjenige, der mehr interessante Ressourcen kontrolliert, sondern auch derjenige, der das geringere Interesse an den Ressourcen hat, die der andere unter Kontrolle hat» (Esser, 1999a, S. 347).

Während Kooperation also in einer Bündelung von Gütern zur Erreichung gemeinsam interessierender Ressourcen mündet und oft mit einer «Integration [der] Orientierungen und des Handelns und schließlich des gesamten sozialen Systems, in das sie eingebettet sind» (Esser, 1999b, S. 146) einhergeht, liegt ein Konflikt dann vor, wenn die Akteure die Kontrolle über die gleichen Ressourcen beanspruchen, mit denen sie ein starkes Interesse verbinden. Von einem Kontroll-Konflikt (oder Konkurrenz) kann laut Esser (Esser, 1999a, S. 348) dann gesprochen werden, wenn die Akteure um die Kontrolle einer Ressource streiten, und von einem Interessen-Konflikt dann, wenn sie mit der Ressource, deren Kontrolle sie inne haben, divergierende Interessen verbinden. Im letzteren Fall streiten die Akteure also um die Bewertung der Ressource.

Die grundsätzliche Unausgeglichenheit der Verteilung von Interessen und Kontrolle, welche Kooperation, aber auch Konflikte erzeugt, wird auf der gesellschaftlichen Ebene in erster Linie mittels Institutionen, d. h. «in den Erwartungen

\footnotetext{
${ }^{7}$ Autonomie und Dependenz bezeichnen Grenzfälle der Verteilung von Interesse und Kontrolle: Autonomie ist gegeben, wenn ein Akteur über alle interessierenden Ressourcen auch die vollständige Kontrolle besitzt. Im Falle der Dependenz hat ein Akteur A die Güter unter Kontrolle, die einen Akteur B interessieren, dieser selber kontrolliert aber keine Ressourcen, die Akteur A interessieren (vgl. Esser, 1999a, S. 343-347).
} 
der Akteure verankerte und sozial verbindliche Regeln des Tuns» (Esser, 2000b, S. 7), reguliert.

Verfassungen, Normen, Regeln, Konventionen, das Recht ganz allgemein sowie der Staat mit seinem Verwaltungsstab und dem Gewaltmonopol - für alle Fälle - gehören zu solchen Institutionen. [...] Wie auch immer aber Institutionen entstanden sind: Ihr Bestehen ist niemals abgesichert. Immer stehen sie in Konkurrenz zu anderen Regelungen und Vorschriften. Dekrete, Verträge und Gewohnheiten können sich ändern. Und immer kostet ihre Durchsetzung etwas: Die Abweichungen müssen sozial kontrolliert und sanktioniert werden, die Mitglieder der Gesellschaft müssen entsprechend sozialisiert werden und die einschränkenden Wirkungen und Zumutungen jeder Institution müssen ertragen werden. (Esser, 1999a, S. 356-357)

Genauso wie einem Akteur in einer sozialen Situation Handlungsmöglichkeiten eröffnet werden, werden seine Handlungsalternativen auch eingeschränkt. Restriktionen für die Selektion von Alternativen ergeben sich für die Akteure aber nicht nur in Form von institutionellen Vorgaben, sondern auch in «natürlicher» Hinsicht, indem sie nur begrenzt über Energie, räumliche, zeitliche und kognitive Ressourcen verfügen, emotional nur begrenzt belastbar sind und auf den Umstand Rücksicht nehmen müssen, dass nicht gleichzeitig zwei Handlungen ausgeführt werden können (vgl. Esser, 1999a, S. 220)

Esser will die Beziehung zwischen kollektiven Phänomenen und individuellen Handlungen umfassend rekonstruieren und konzipiert dazu sein $u$. a. an Colemans (1990, S. 1-23) Makro-Mikro-Makro-Schema orientierten «Modell der sozialen Erklärung» (vgl. Esser, 1999a, S. 91-111; Esser, 1999b, S. 15-20), welches aus drei Erklärungsschritten ${ }^{8}$ besteht.

Bei der Verbindung zwischen der sozialen Situation und dem individuellen Akteur spricht er von der «Logik der Situation» (Makro-Mikro-Verbindung). Bevor der Akteur überhaupt handeln kann, muss sein kognitives System die Situation erfassen. In Anlehnung auf das auf Minsky (1975) zurückgehende kognitionspsychologische Konstrukt des Frames als «a data-structure for representing a stereotyped situation» (S. 211) spricht Esser (2001, S. 261-264) davon, dass ein Framing der Situation, ein Orientierungsprozess, vorgenommen werde, mit dessen Hilfe ein Akteur die Handlungsbedingungen erfasse, Zielprioritäten festlege und sich in der sozialen Situation hinsichtlich ihrer Opportunitäten und Restriktionen verorte. Demnach wird ein mentales Modell aktiviert, das hinsichtlich der wahrgenommenen Situation die beste Passung aufweist und das gleichzeitig «in typisierter Form die spezielle inhaltliche Definition der Situation,

${ }^{8}$ Diese bilden in der soziologischen Untersuchung gleichzeitig auch die Analyseschritte. 
insbesondere aber das Oberziel, um das es in der betreffenden Situation geht» enthält, wobei «das Oberziel ... den funktionalen, kulturellen oder normativen Code des Frames» festlegt (Esser, 2001, S. 263, Hervorhebungen im Original). Ebenso wird laut Esser in dieser Phase geprüft, ob bereits ein passendes Skript, ein mentales Modell einer typischen Handlungssequenz (vgl. Schank \& Abelson, 1977), für den entsprechenden Frame der Situation im eigenen Repertoire verfügbar ist. Liegt ein Match zwischen den in der Situation wahrgenommenen und den im mentalen Modell erwarteten Objekten vor, kommt es zu keinen kognitiven Aktivitäten höheren Bewusstheitsgrades und die im Modell angelegte Handlungssequenz gelangt zur Ausführung. Esser erklärt so insbesondere normbefolgendes Handeln, wie es im Rollenkonzept des Strukturfunktionalismus angelegt ist: Individuen halten sich dann an die Selektionen, die innerhalb des Frames überhaupt möglich sind. Der Akteur kann nun allerdings immer auch - «finding und resourceful, wie er von seiner Natur her ja im Prinzip ist» (Esser, 1999a, S. 247) - nach Handlungsalternativen suchen, für die diese Restriktionen nicht gelten. Im Einklang mit der Psychologie des Problemlösens, wo von einem Problem dann gesprochen wird, wenn sich der Handelnde dem Ungenügen seiner Handlungs-, seiner Operationspläne oder seiner Wahrnehmung hinsichtlich eines Handlungs-, Operations- oder Verstehensziels bewusst wird (vgl. Aebli, 1994, S. 13-19) postuliert er, dass in einem solchen Fall - einem Mismatch also - Informationsverarbeitungsprozesse eingeleitet würden und je nach Einschätzung der Bedeutsamkeit, des erforderlichen Aufwands und der zur Verfügung stehenden Opportunitäten unterschiedlich elaborierte, aufwändige und systematische Heuristiken zur Interpretation und Anpassung des Situationsmodells sowie des Handlungsprogramms selektiert würden. Wie reflektiert dies geschieht, unterliegt nach Ansicht Essers also ebenfalls der Selektionsregel der Erwartungs-Wert-Theorie: «Die Erwartungen und Bewertungen beziehen sich auf die Sicht der Situation aus der Perspektive des Akteurs. Die subjektiven Erwartungen und Bewertungen von Konsequenzen spiegeln die Alltagstheorien und die - immer institutionell vermittelten - grundlegenden Ziele der Menschen wider» (Esser, 1999a, S. 247) ${ }^{9}$.

Die Selektionsregel der Erwartungs-Wert-Theorie stellt aber insbesondere auch bei der «Logik der Selektion» das Kernelement dar. In diesem zweiten Schritt der soziologischen Erklärung geht es darum zu rekonstruieren, warum die Akteure

\footnotetext{
${ }^{9}$ Esser versteht Entscheidungen prinzipiell nicht als willentlich und bewusst vorgenommene Entschlüsse. Kron (2004, S. 190) erläutert dies so: «Esser dehnt den Entscheidungsbegriff soweit aus, dass nur noch die Auswahl selbst die Entscheidung trägt. Findet eine Auswahl statt, wurde entschieden. Nur so kann auch z. B. der Organismus ,entscheiden', welche Informationen er abspeichert und welche nicht, etwa wenn Verhaltensweisen verstärkt werden».
} 
so handeln, wie sie es konkret tun (Mikro-Mikro-Verhältnis zwischen Akteur und Handlung). Die in der Situationsanalyse erkannten Handlungsalternativen werden demnach nun hinsichtlich ihrer Konsequenzen evaluiert und immer diejenige ausgewählt, die subjektiv gesehen die «beste Kosten-Nutzen-Bilanz» (Jäger \& Meyer, 2007, S. 112) aufweist, d. h. den größten zu erwartenden Nutzen bzw. die geringsten Kosten nach sich zieht. Rationalität liegt nach Esser bei der Situationswahl (Framing) und bei der Handlungswahl vor, nicht aber in ihrem Ergebnis. Menschen unterlaufen Fehler beim Abschätzen der Handlungskonsequenzen - besonders beim sozialen Handeln, wo nach dem Prinzip der doppelten Kontingenz (vgl. Parsons \& Shils, 1954, S. 16) das eigene Handeln und das Handeln der anderen Akteure wechselseitig aufeinander bezogen sind. Sicherheit bei der Entscheidung (d. h. eine Eintrittswahrscheinlichkeit $\mathrm{p}$ $=1$ oder $\mathrm{p}=0)$ genauso wie der Fall des Risikos $(0<\mathrm{p}<1)$, wo die Wahrscheinlichkeit des Eintretens zwar nicht sicher, aber präzise feststeht, dürften in sozialen Situationen kaum je gegeben sein. Wahrscheinlicher ist Ungewissheit $(\mathrm{p}=$ ?), vor allem aber Ambiguität, d. h. «die Streuung der Einschätzung des Risikos um ein bestimmtes p als Mittelwert der Erwartungen» (Esser, 1999b, S. 255). Hierin unterscheidet sich das Modell von Esser denn auch wesentlich von demjenigen des klassischen ökonomischen Rational Choice-Ansatzes, dem das Menschenbild des homo oeconomicus zugrunde liegt: sichere Erwartungen aufgrund perfekter Information ist im Sozialen genauso unrealistisch wie die Annahme stabiler persönlicher Bewertungsmuster (stabile Präferenzordnungen) ohne Rücksicht auf situative Bedingungen (vgl. Diefenbach, 2009, S. 242; Esser, 1999a, S. 236-237; Fend, 2006, S. 151). Wie bei der Situationseinschätzung, wo höhere Bewusstseinsprozesse im Sinne einer Kosten-, Nutzen- und Wahrscheinlichkeitskalkulation nur bei nicht-genügenden Frames und Skripts einsetzen, findet eine reflektierte Ziel-Mittel-Einschätzung bei der Handlungswahl lediglich dann statt, wenn vom Akteur Probleme wahrgenommen werden. Ansonsten laufen die Entscheidungsprozesse hoch automatisiert ab (zum Konzept der Habits als Mittel zur Reduktion des Reflexionsaufwands, vgl. Esser, 1990).

Mit dem dritten Schritt seines Erklärungsmodells, der Logik der Aggregation, stellt Esser schließlich die Verbindung zwischen dem Handeln der Akteure und den kollektiven Phänomenen, die sich daraus ergeben, her (Mikro-MakroVerbindung). Mittels «Transformationsregeln» (vgl. Esser, 2000a, S. 13-29) modelliert Esser, wie aus dem Zusammenwirken der individuellen Handlungen und Handlungsfolgen gesellschaftliche Phänomene bzw. kollektive Zustände wie z. B. die Maturand*innen-Quote, der Bildungserfolg von Jugendlichen aus privilegierten Schichten, die Organisation der schulischen Grundausbildung im Kanton Zürich etc. emergieren. 
Für die vorliegende Studie, die das wechselseitige Handeln der Individuen, die inneren Prozesse und dessen Effekte auf der Mikroebene fokussiert, kann die Erläuterung des Erklärungsmodells von Esser an dieser Stelle zu einem Schluss kommen. Im Folgenden wird nun auf der Grundlage des Erarbeiteten eine Definition des Begriffs «Elterliche schulbezogene Unterstützung» vorgenommen.

\subsection{Erläuterung des Begriffs der elterlichen schulbezogenen Unterstützung}

Wie können die Kernpostulate der Handlungstheorie Essers nun für die Modellierung der sozialen Situation nutzbar gemacht werden, in der sich die Eltern mit ihrem Kind und dessen Klassenlehrkraft ${ }^{10}$ in der Phase vor dem Übertrittsentscheid befinden?

Die vorliegende Studie folgt dem Menschenbild, welches Esser im Anschluss an Arbeiten von Lindenberg (1985) seiner Theorie zugrunde legt. Es ist dies das Konzept des «Resourceful-Restricted-Expecting-Evaluating-MaximizingMan»(RREEMM-Modell): Übersetzt auf die hier interessierenden Hauptakteure, die Eltern, bedeutet das,

a) dass sie ihre Interessen, Aspirationen und Ziele, ihre kognitiven Fähigkeiten und affektiv-motivationalen Dispositionen sowie zeitlichen und materiellen Ressourcen in die jeweiligen sozialen Situationen mit dem Kind und der Klassenlehrkraft einbringen,

b) dass sie grundsätzlich resourceful, das heißt «findig, kreativ, reflektiert und überlegt» (Esser, 1999a, S. 238) Handlungsmöglichkeiten (Opportunitäten) in der jeweiligen Situation zu erkennen suchen,

c) dass sie aber immer zugleich auch situative Einschränkungen in Form von Normen, institutionellen Regeln und Gesetzen sowie in Form von Erwartungen und begrenzten Ressourcen ihrer Koakteure Kind und Lehrkraft vorfinden bzw. vorzufinden glauben (restricted),

d) dass sie «immer eine ,Wahl` haben» (Esser, 1999a, S. 238, Hervorhebung im Original), d. h. routiniert-habitualisiert auf die Situation einwirken oder

\footnotetext{
${ }^{10}$ Die Klassenlehrkraft ist im Übertrittsverfahren der Volksschule des Kantons Zürich in der Regel die Hauptansprechperson der Eltern. Der Einfachheit halber wird im Folgenden deshalb jeweils sie erwähnt, obwohl immer auch weitere schulische Akteure der Mikro- und Mesoebene, wie z. B. weitere Fach- und Förderlehrpersonen, Mitglieder der Schulleitung und Schulbehörden, je nach Phase des Verfahrens mal mehr, mal weniger direkt mitbeteiligt sind.
} 
reflektiert verschiedene Handlungsalternativen abwägen können und sich ggf. auch für ein Nichts-Tun entscheiden können,

e) dass diese Selektionen immer aus dem kognitiven Zusammenspiel von Konsequenzerwartungen (expecting) und Bewertungen der Handlungsalternativen (evaluating) bestehen, wobei die Eltern schließlich mit Blick auf die eigenen Ziele diejenigen wählen, welche die günstigsten Ergebnisse erwarten lassen (maximizing).

Auf dieser Grundlage und mit Blick auf die eingangs gemachten Aussagen zu den zwei Bedeutungskernen des Terminus «unterstützen» - jemandem materiell oder mit Rat und Tat behilflich sein sowie sich für jemandes Angelegenheit bei jemandem andern einsetzen - definiert sich elterliche schulbezogene Unterstützung in der vorliegenden Studie folgendermaßen:

Elterliche schulbezogene Unterstützung bezeichnet ein an den Zielen und Bedingungen des Schulsystems orientierter Ressourceneinsatz der Eltern, der darauf gerichtet ist, die von ihnen als bedeutsam beurteilten Kompetenzen und Laufbahnschritte beim Kind zu fördern und/oder das Denken und Handeln der schulischen Akteure im Interesse des Kindes zu optimieren.

Die Definition bestimmt elterliche Unterstützung als Ressourcenallokation der Eltern für den schulischen Lebensbereich ihrer Kinder und unterscheidet, wie dies seit Jahren in der angelsächsischen parental involvement-Forschung gebräuchlich ist, zwischen einer Unterstützung zuhause («involvement based at home») und einer solchen in der Schule («involvement based at school») (Pomerantz et al., 2007, S. 374). Elterliches schulbezogenes Unterstützen wird hier somit als mehrdimensionales domänenspezifisches Handeln der Eltern bestimmt, das sich einigermaßen klar von anderen kindbezogenen Handlungsfeldern trennen lässt und auf Verhaltensänderungen bei den Mitakteuren Kind und Klassenlehrkraft (sowie weiterer von ihnen als bedeutsam erachteten Funktionsträgern des Schulsystems) abzielt. Die intendierte Wirkung dieses sozialen Handelns besteht mit anderen Worten in einer irgendwie gearteten, subjektiv von den Eltern als Optimierung bewerteten Veränderung des Denkens und Handelns der beiden Akteure Kind und Klassenlehrkraft. Je nach ihren persönlichen Werten, Zielen, Aspirationen und selbsteingeschätzten Kompetenzen, aber auch je nach wahrgenommenen situativen Handlungsspielräumen sowie interpersonalen Erwartungen und Forderungen der beiden primären Handlungspartner, obliegt es grundsätzlich den Eltern zu entscheiden, welche Ressourcen sie in welcher Form und in welchem Umfang in welche aus ihrer Sicht lohnenswerten Aspekte in den beiden Handlungssphären Elternhaus und Schule investieren wollen. 


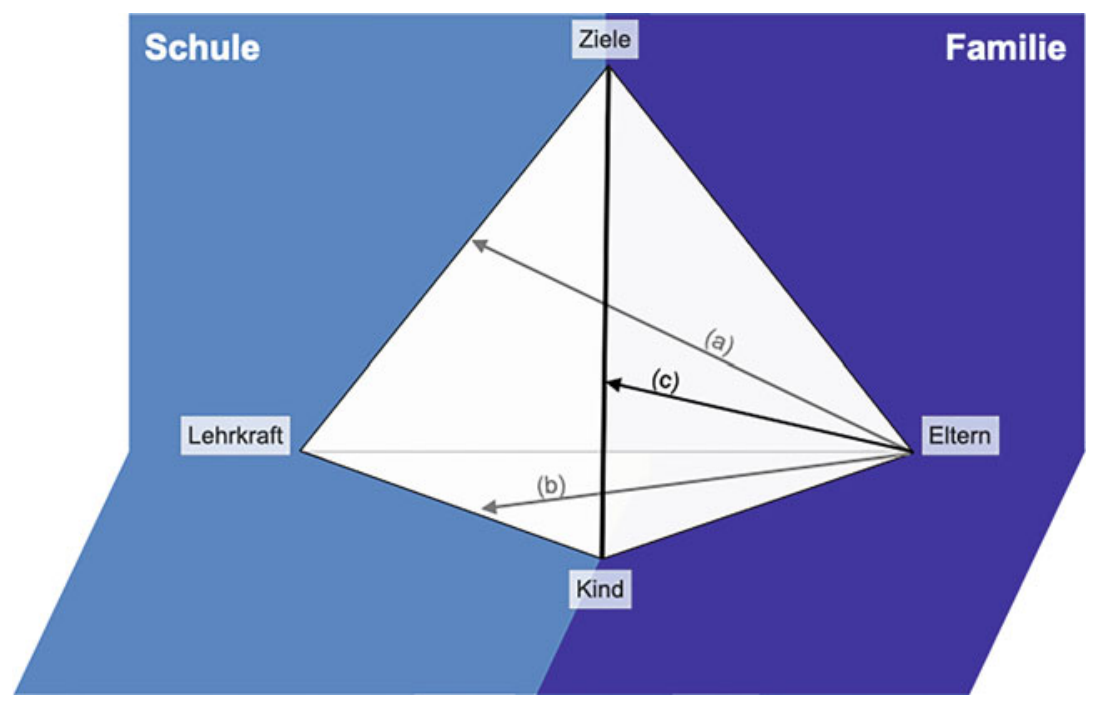

Abbildung 2.1 Strukturmodell elterlichen schulbezogenen Unterstützungshandelns

Abbildung 2.1 illustriert die Stoßrichtungen des möglichen Ressourceneinsatzes der Eltern, indem es die Denkfigur des Didaktischen Dreiecks (z. B. Bönsch, 2006, S. 149-159; Fuchs, 2008, S. 1026-1027; kritisch bei Gruschka, 2002, S. 87; prominent bei Reusser, 2008), die seit langem zur Beschreibung des «Zusammenspiel[s] der drei wichtigsten Faktoren didaktischer Prozesse des Unterrichts» (Schröder, 2001, S. 75) verwendet wird ${ }^{11}$, um den Pol der Eltern erweitert und so drei Ansatzpunkte für das elterliche Handeln in den beiden Handlungssphären Schule (Interaktion mit der Klassenlehrkraft) und Familie/Elternhaus (Interaktion mit dem Kind) sichtbar werden lässt (s. die Graphen a, b und c). Das Modell hat eine primär heuristische Funktion. Der hohe Grad der Idealisierung der tatsächlichen Verhältnisse wird nicht zuletzt daraus ersichtlich, dass die anvisierten Bildungsziele der drei Akteure in Wirklichkeit nie deckungsgleich sind. Daneben birgt das Modell aber auch eine normative Funktion: So verweist gerade die

\footnotetext{
${ }^{11}$ Das Didaktische Dreieck wird gewöhnlich von den drei Kategorien Lernende/r, Lehrkraft sowie Bildungsgegenstand bzw. Bildungs- und Erziehungsziele gebildet.
} 
im Modell gewählte Darstellung gemeinsam geteilter Bildungs- und Erziehungsziele $^{12}$ auf die Wichtigkeit einer möglichst hohen Ziel- (und Mittel-)Kongruenz zwischen den Eltern und den Lehrkräften im Sinne «konsistenter Botschaften an das Kind über die Bedeutsamkeit von Bildungszielen» (Epstein, 1990, S. 100, Übersetzung E.S.) und einer dafür notwendigen Partnerschaftlichkeit zwischen beiden Akteuren (vgl. Ho \& Vasarik Staub, 2019; Neuenschwander, 2013; Neuenschwander et al., 2005; Sacher, 2014; Stange, 2012) (vgl. Kapitel 3).

Die wenigen empirischen Studien, die im deutschsprachigen Raum zur Thematik der Zusammenarbeit der beiden Sozialisationsinstanzen existieren, deuten darauf hin, dass es dann zu einem partnerschaftlichen Verhältnis zwischen den Lehrkräften und den Eltern kommt, wenn die Beziehung insbesondere von wechselseitiger Informiertheit, von kindbezogenen Abstimmungen von Erwartungen und Wahrnehmungen, von gelingender Koordination pädagogischer Maßnahmen sowie von gegenseitigem Vertrauen geprägt ist, wobei es aus der Sicht beider Akteure die Lehrkräfte sind, von denen die Initiative zur Zusammenarbeit erwartet wird (vgl. Neuenschwander et al., 2005, S. 182-207; Sacher, 2006). Ein Umstand, der damit zusammenhängen dürfte, dass der elterliche Beitrag eben lediglich als unterstützend in einem Handlungsfeld wahrgenommen wird, das primär von den schulischen Akteuren im Rahmen der institutionellen Vorgaben gestaltet wird.

\subsubsection{Elterliche Unterstützung in der Schule des Kindes}

«Involvement based at school» bedingt denn auch die direkte Kommunikation der Eltern mit schulischen Akteuren (Pomerantz et al., 2007, S. 374). Der Graph (a) in Abbildung 2.1 repräsentiert den Versuch der Eltern im Rahmen persönlicher Gespräche, auf die Zieldefinition von Lehrkräften und deren Ressourcenallokation zugunsten des Kindes Einfluss zu nehmen. Lehrkräften obliegt es, Zielvorgaben des Bildungssystems - «rechtliche Vorgaben, Lehrpläne, Prüfungsanforderungen und [einzel-]schulische Vereinbarungen» (Fend, 2008, S. 37) - in ein Angebot an die Schülerinnen und Schüler zu übersetzen, das sich laut Reusser (2008, S. 225) durch hohe «personale und kulturelle Signifikanz der Inhalte», durch

\footnotetext{
${ }^{12}$ Bildungs- und Erziehungsziele können mit Klieme et al. (2007, S. 20) als «relativ allgemein gehaltene Aussagen darüber [verstanden werden], welche Wissensinhalte, Fähigkeiten und Fertigkeiten, aber auch Einstellungen, Werthaltungen, Interessen und Motive die Schule vermitteln soll». Bildungsziele geben allerdings nur «recht generelle Erwartungen wieder, [...] die in Form von Kompetenzanforderungen» (S. 21) auf den verschiedenen Ebenen des Bildungssystems konkretisiert werden müssen (nationale Bildungsstandards, um Kompetenzmodelle ergänzte Lehrpläne, angepasste Diagnose- und Evaluationsmittel etc.).
} 
eine hohe «Lernaufgabenqualität» sowie eine kohärenz- und interessestiftende «Lehrstofforganisation» auszeichnet. Mit Blick auf die Allokations- und Selektionsfunktion der Schule (vgl. Fend, 2006, S. 50) obliegt es ihnen ferner, reflektierte und transparente Diagnose-, Prüf- und Auswahlverfahren zu gestalten, die sich an wissenschaftlichen Gütekriterien und den Vorgaben des jeweiligen Übertrittsverfahrens orientieren (z. B. Pohlmann, 2009; Schrader, 2014; Terhart, 2014). Je nach Opportunitätsstrukturen, die das jeweilige Schulsystem bzw. die schulischen Akteure mit ihren rekontextualisierten Verfahren den Eltern anbieten (Anzahl und Art der Gesprächsgefäße, Einfachheit des Zugangs zu Lehrkräften bei Gesprächsbedarf; vgl. Abschnitt 3.2), und je nach eigenen motivationalen Orientierungen (Selbstwirksamkeitsüberzeugungen, Wertüberzeugungen und Aspirationen; vgl. Abschnitt 4.2.1.2), können die Eltern im Rahmen von Gesprächen ${ }^{13}$ mit der Klassenlehrkraft namentlich vor einem schulischen Übergang versuchen, ein klareres Bild von deren anvisierten Lern- und Leistungszielen, deren Unterrichtsprogramm, deren Kriterien der Leistungsbeurteilung sowie von deren Beurteilung des Kompetenzstandes des eigenen Kindes zu gewinnen. Eltern können in solchen Gesprächen ihre eigenen Standpunkte und Sichtweisen einbringen, ihre Ziele und Aspirationen deutlich machen und so die Wahrnehmung der Lehrkraft bezüglich ihres Kindes in ihrem Sinne zu optimieren versuchen.

Da es sich bei Unterricht und Schule aus der Sicht der Eltern um ein Exosystem ${ }^{14}$ handelt, an dem sie laut Sacher (2012, S. 235) «in peripheren Bereichen» wie Schulfeiern, Klassenausflügen u. ä., «kaum aber in größerer Nähe zum ,Kerngeschäft ${ }^{\star}$ - im Regelunterricht, im Nachhilfe- und Förderunterricht oder bei der schulischen Hausaufgabenbetreuung» direkt partizipieren, wird es ihnen lediglich in Gesprächen mit den Lehrkräften gelingen, formativ auf die Gestaltung der «Interaktions-, Beziehungs- und Lernhilfequalität» (Reusser, 2008, S. 225) zwischen den Lehrkräften und ihrem Kind (bzw. dessen Klasse) Einfluss zu nehmen, wie von Graph (b) in Abbildung 2.1 symbolisiert. Eltern können sich direkt bei den Lehrkräften über instruktions- und beziehungsbezogene Aspekte informieren,

\footnotetext{
${ }^{13}$ Eltern nicht nur versuchen, im Rahmen von Gesprächen die Zielsetzungen einzelner Lehrkräfte ihres Kindes auf der Mikroebene mitzugestalten, sondern können je nach Gestalt des jeweiligen Bildungssystems im Rahmen von Elterngremien oder im Rahmen von Schulaufsichtsbehörden und politischer Gremien auf der Meso- und Makroebene versuchen, Einfluss auf Entscheidungsprozesse, Zielsetzungen und Mittelallokationen zu nehmen. Epstein (1990, S. 114) bezeichnet solcherlei elterliches Engagement als «involvement in governance and advocacy».

14 «Unter Exosystem verstehen wir einen Lebensbereich oder mehrere Lebensbereiche, an denen die sich entwickelnde Person nicht selbst beteiligt ist, in denen aber Ereignisse stattfinden, die beeinflussen, was in ihrem Lebensbereich geschieht, oder die davon beeinflusst werden.» (Bronfenbrenner, 1993, S. 42)
} 
um aus erster Hand aus deren Perspektive - und nicht lediglich aus derjenigen des Kindes - Aufschluss über deren handlungsleitenden Maximen und Überzeugungen sowie über deren Sicht auf konkrete unterrichtliche Ereignisse zu gewinnen. Andererseits können sie auch versuchen, die kognitiven, sozialen und emotionalen Bedürfnisse ihres Kindes zu verbalisieren, seine Wahrnehmung von Ereignissen in Unterricht und Schule zu schildern und so in seinem Interesse auf die kindbezogene Kommunikation, Beziehung sowie Lernunterstützung und -beratung der Lehrkraft (vgl. Reusser, 2008, S. 228-229) optimierend Einfluss zu nehmen.

Wie die Eltern bei diesen Einflussnahmen konkret vorgehen, ist bisher weitgehend unerforscht. Nimmt man die vorderhand inkonsistente Befundlage ${ }^{15}$ zur Forschung um das parental involvement based at school und um die «Bildungsund Erziehungspartnerschaft» (school family partnership) (Sacher, 2014; Sacher, Berger \& Guerrini, 2019) in den Blick, so zeigt sich vor allem deutlich, dass primär Eltern mit hohem Bildungs- und Sozialkapital von sich aus das Gespräch zu den Lehrkräften suchen und sich aktiv in der Schule einbringen (vgl. Abschnitt 4.2.1), indem sie sich z. B. in den Elterngremien engagieren (vgl. Sacher, 2012). Eltern mit niedrigem sozioökonomischem Status und/oder Alleinerziehende sind weniger präsent und scheinen auf die Initiierung der Kommunikation durch die Schule angewiesen zu sein (vgl. Sacher, 2012; Sheldon \& Epstein, 2005). Ferner gibt es Hinweise dafür, wonach es Eltern aus bildungsnahem Milieu tatsächlich gelingt, direkten Einfluss auf die Leistungsbeurteilung

\footnotetext{
${ }^{15}$ Die mitunter widersprüchliche Befundlage liegt vor allem daran, dass bisher nur wenige längsschnittliche Studien vorliegen. Bei Kontrolle der (Vor-)Leistungen des Kindes deuten bestehende längsschnittliche Studien darauf hin, dass das (verstärkte) elterliche Engagement in der Schule prädiktiv für einen Leistungszuwachs zumindest in der Primarstufe ist (vgl. Dearing, Kreider, Simpkins \& Weiss, 2006; Grolnick, Kurowski, Dunlap \& Hevey, 2000; Gutman \& Eccles, 1999; Izzo, Weissberg, Kasprow \& Fendrich, 1999; Simpkins et al., 2015a). Pomerantz et al. (2007, S. 379) kommentieren: «definitive conclusions about the causal role of parents' involvement on the school front await experimental designs manipulating parents' involvement». Bezüglich bestehender querschnittlicher Studien beklagen Fan und Chen (2001, S. 17) und Wilder (2014, S. 378) vor allem die uneinheitlichen, vielfältigen Operationalisierungen von parental involvement generell sowie besonders von spezifischen Unterstützungskomponenten. Ebenso weisen sie darauf hin, wie unterschiedlich die Zielvariable Leistung in den zugrundeliegenden Studien erhoben und gemessen werden (Noten in Kernfächern, Notenschnitt im Zeugnis, standardisierte Leistungstests, Ratings von Lehrkräften etc.) (vgl. auch Sacher, 2012). In den Metaanalysen bei Jeynes (2005, 2007) finden sich keine Effekte elterlicher Partizipation in der Schule auf die Leistungsentwicklung der Kinder, in den Metaanalysen von Hill und Tyson (2009) ist ein moderat positiver Effekt zu verzeichnen, Hattie (2009, S. 68) berichtet auf der Basis einer Metaanalyse von Rosenzweig (2001) von geringen Effektstärken von je $d=0.14$ für participation in school activities und communication with school and teachers.
} 
der Lehrkräfte auszuüben, insofern als sich je nach sozialem Hintergrund des Elternhauses deutliche Unterschiede zwischen Zeugnisnoten und den Ergebnissen standardisierter Leistungstests zugunsten der Kinder aus privilegiertem Elternhaus belegen lassen (vgl. Abschnitt 4.1.1). So zeigen auch Neuenschwander und Kolleg*innen (2005, S. 235) in einer groß angelegten Studie im Kanton Bern, dass Kinder bei gleichen Testleistungen von den Lehrkräften besser benotet wurden, wenn die Eltern hohe Bildungserwartungen aufwiesen (Item: Erwartung höchster Schulabschluss) und wenn diese gute Leistungen des Kindes internal auf dessen Fähigkeiten und Interessen attribuierten. Umgekehrt erhielten Lernende bei gleichen Testergebnissen schlechtere Benotungen durch die Lehrkraft, wenn deren Eltern gute Noten mit geringer Aufgabenschwierigkeit - also mit einer external stabil unkontrollierbaren Ursache - begründeten. Wie die Autor*innen festhalten, könne über die Wirkmechanismen allerdings nur spekuliert werden: «Vielleicht werden diese Erwartungen und Ursachenzuschreibungen von den Eltern direkt oder [auch nur] indirekt über das Kind kommuniziert und von der Lehrperson bei der Benotung berücksichtigt» (Neuenschwander et al., 2005, S. 235). Trotz fehlender empirisch abgesicherter Aussagen über die Wirkpfade und deren Effektstärken, erscheint die These plausibel, wonach erfolgsorientierte Eltern, die selber oft einen hohen beruflichen Status aufweisen (vgl. Neuenschwander, Vida, Garrett \& Eccles, 2007; Shumow \& Miller, 2001), ihre kindbezogenen Aspirationen, Erwartungen und günstigen Attributionsmuster in Gesprächen mit den Lehrkräften genauso zum Ausdruck bringen, wie sie dies auch ihrem Kind gegenüber tun (vgl. Neuenschwander et al., 2005, S. 243). Es ist sodann ebenso plausibel, dass dieser Umstand das Kind in den Augen der Lehrkraft leistungsmäßig in einem besseren Licht erscheinen lässt und sich dies im Sinne des Pygmalioneffekts (vgl. Rosenthal \& Jacobson, 1968) nicht nur auf die Leistungsbewertung der Lehrkraft, sondern auch auf deren Lernbegleitung mit all ihren Facetten positiv auswirkt ${ }^{16}$. Ähnliches vermutet Jeynes, der in zwei seiner Metaanalysen $(2003,2005)$ den Einfluss verschiedener Größen elterlichen Engagements auf unterschiedliche Leistungsmaße (Notendurchschnitt im Zeugnis, standardisierte Leistungstests sowie Ratings der Klassenlehrkräfte bezüglich Lern- und Leistungsverhalten und motivationalen Orientierungen) bei amerikanischen Schüler*innen der Primarstufe und Sekundarstufe I untersuchte. Wie sich zeigte, resultierte zwar bei allen Arten der Leistungsmessung ein signifikanter positiver Effekt, der von elterlichen schulbezogenen Unterstützungen (general parental involvement) ausging, allerdings fiel dieser Effekt in beiden Studien am schwächsten bei den standardisierten Leistungstests und (erwartungsgemäß) am stärksten bei den Ratings der Lehrkräfte

\footnotetext{
${ }^{16}$ Kritisch zum Pygmalioneffekt bzw. zu self-fulfilling prophecies: Jussim und Harber (2005)
} 
aus. Von diesen Befunden ausgehend, weist Jeynes (2003) auf die möglicherweise bedeutende Rolle hin, die eine positive Wahrnehmung der Lehrkräfte des elterlichen Handelns in der Schule - die Zeit, die diese dafür einsetzen können und wollen, die geteilten Ziele und Werte, die sichtbar werden - auf ihre Wahrnehmung und Einschätzung des Kindes haben könnte:

It is likely that teacher ratings may be affected by teacher perceptions of the level of cooperation exhibited by the child and the family as a whole. To the extent that parental involvement may be the major component of that perceived cooperation, teachers may view children and their families more positively as a result of that perceived cooperation. $[\ldots]$ Teachers $[\ldots]$ may feel inclined to reward good motives by the child and the family. Teacher ratings are more likely than other academic measures to reflect (a) a positive relationship between the parent and the teacher; (b) a sense of teamwork between the parent and the teacher, due to increased communication between the two; and (c) an acknowledgment by the teacher of parental efforts. (Jeynes, 2003, S. 213-214)

Die Chancenungleichheit, die aus den skizzierten Zusammenhängen für Kinder aus weniger privilegierten Elternhäusern manifest wird - «Zusammenarbeit von Eltern mit Lehrpersonen verstärkt faktisch [geradezu] die Chancenungleichheit» (Neuenschwander et al., 2005, S. 242), weil offenbar vor allem Kinder aus bildungsnahem Milieu profitieren -, wird in Abschnitt 3.1 bezüglich schulischer Übergänge unter dem Stichwort «Herkunftseffekte» noch genauer erörtert werden.

Die in Abschnitt 4.1 näher erläuterte Kapitalientheorie Bourdieus $(1984,1998)$ und deren Postulat kommunikativer Passungsprobleme, die sich für Familien mit geringem sozialen und kulturellen Kapital in der «mittelschichtsorientierten» Schule (vgl. Rolff, 1997, S. 134) ergeben, bildet dahingegen einen Ansatz zur Erklärung der Befunde in der gleichen Studie (Neuenschwander et al., 2005, S. 229-233), wonach Eltern aus tieferen Schichten und/oder mit Migrationshintergrund sich zwar sehr interessiert an der Zusammenarbeit mit der Schule zeigen und ebenso eine generell höhere Zufriedenheit mit deren Informationspolitik bekunden als Eltern aus höheren Schichten, für eine aktive Partizipation aber offenbar auf eine «Willkommenskultur» der Schule (Sacher, 2014) angewiesen sind. So scheinen Eltern aus höheren Schichten über höhere Erwartungen hinsichtlich der Häufigkeit und der Gestaltung der Informationen über Unterricht und Erziehung in der Schule zu verfügen, sich in ihrem sozialen Netz auch anderweitig die notwendigen Informationen und Hilfestellungen verschaffen zu können und die dafür relevanten Kommunikationstechniken zu beherrschen, die sie bei Bedarf angstfreier auf die Lehrkräfte zugehen lassen. Schulen und Lehrkräfte 
sind demnach aufgefordert, im Zeichen der Chancengerechtigkeit selbstinitiiert den Kontakt besonders zu Eltern sozial benachteiligter Kinder zu suchen.

\subsubsection{Häusliche schulbezogene Unterstützung der Eltern}

Bezüglich der Effekte auf das Lernen und Leisten sowie das Sozialverhalten von Schülerinnen und Schülern ist vor allem gesichert, dass vom Unterstützungshandeln im Elternhaus - «involvement based at home» - die bedeutend stärkeren Wirkungen ausgehen als von den oben erläuterten relativ unterrichtsfernen elterlichen Aktivitäten in der Schule (vgl. Fan \& Chen, 2001; Jeynes, 2007; Sheldon \& Epstein, 2005; Shumow \& Miller, 2001). Dies leuchtet auch unmittelbar ein, zieht man in Betracht, dass das Kind in diesem Mikrosystem ${ }^{17}$ der direkte Interaktionspartner der Eltern ist, mit dem sie die konkreten unterrichtsund schulbezogenen Angelegenheiten erörtern und bearbeiten können. Wie der Graph $\mathrm{c}$ in Abbildung 2.1 signalisiert und es in der obigen Begriffsdefinition zum Ausdruck kommt, richtet sich das schulbezogene häusliche Handeln der Eltern darauf, die Lernprozesse des Kindes so zu regulieren, dass dieses die von ihnen als zentral erachteten schulischen Bildungsziele erreicht. Die Beschränkung auf die schulische Domäne grenzt denn auch dieses elterliche Handeln von ihrem übrigen erzieherischen Handeln ab: Während in Erziehungsprozessen immer die Situation vorliegt, dass «Erwachsene versuchen, in den Prozess des Werdens heranwachsender Persönlichkeiten [...] einzugreifen, um Lernvorgänge zu unterstützen oder in Gang zu bringen, die zu Dispositionen und Verhaltensweisen führen, welche von den Erwachsenen als seinsollend oder erwünscht angesehen werden» (Brezinka, 1971, S. 26), sind es hier nur jene Dispositionen und Laufbahnziele im Blickfeld der Eltern, die gemäß ihrer Wahrnehmung eine hohe Passung mit ihren eigenen Bildungszielen sowie denjenigen der Lehrkräfte aufweisen. Namentlich im Kontext von Übertrittsverfahren dürften es die meisten Eltern als wichtig erachten, die Leistungserwartungen der schulischen Akteure zu kennen und mit dem Kind auf die Erfüllung derselben hinzuarbeiten. Wie in Kapitel 3 noch näher zu erörtern sein wird, erwächst der Institution Schule mit der gesellschaftlich abgesicherten Selektions- und Allokationsfunktion (vgl. Fend, 2006, S. 50) eine

${ }^{17}$ «Ein Mikrosystem ist ein Muster von Tätigkeiten und Aktivitäten, Rollen und zwischenmenschlichen Beziehungen, die die in Entwicklung begriffene Person in einem gegebenen Lebensbereich mit den ihm eigentümlichen physischen und materiellen Merkmalen erlebt.» (Bronfenbrenner, 1993, S. 38) 
wirkmächtige Ressource, die den von ihr definierten Bildungszielen bzw. Kompetenzanforderungen ein hohes Gewicht gibt und die Eltern und das Kind zu Handlungsanpassungen zwingt (vgl. Pekrun, 2001, S. 86-87).

\subsubsection{Häusliche Kompetenzförderung}

In der obigen Definition elterlichen schulbezogenen Unterstützungshandelns wird mit dem Kompetenzbegriff operiert. Bei aller definitorischen Vielfalt, die mit der «fast inflationären Verwendung» (Arnold \& Lindner-Müller, 2012, S. 229) des Terminus im Kontext der großen internationalen und nationalen Schulleistungsstudien wie PISA, IGLU/PIRLS ${ }^{18}$, DESI ${ }^{19}$ sowie der Einführung von Bildungsstandards (Klieme et al., 2007; Oelkers \& Reusser, 2008) einhergeht, lassen sich Kompetenzen im Kern als «Leistungsdispositionen» (Hartig \& Klieme, 2006, S. 129, Hervorhebung im Original) bezeichnen, die anders als das Konstrukt allgemeine Intelligenz einen klaren Anwendungsbezug aufweisen, somit relativ bereichsspezifisch sind, aber dennoch «über ähnliche Situationen generalisierbar sind» (Hartig \& Klieme, 2006, S. 129). Weinert (2001) hat die Dimensionen Funktionalität, Domänenspezifität und Transferierbarkeit in seine wegweisende Definition eingebracht, indem er darauf verwies, dass es sich bei Kompetenzen um Wissen handle, das hauptsächlich im Rahmen von Problemlöseprozessen aufgebaut bzw. gelernt werde und sich immer wieder in neuen Problemlösesituationen bewähren müsse $\mathrm{e}^{20}$ :

Dabei versteht man unter Kompetenzen die bei Individuen verfügbaren oder durch sie erlernbaren kognitiven Fähigkeiten und Fertigkeiten, um bestimmte Probleme zu lösen, sowie die damit verbundenen motivationalen, volitionalen und sozialen Bereitschaften und Fähigkeiten, um die Problemlösungen in variablen Situationen erfolgreich und verantwortungsvoll nutzen zu können. (Weinert, 2001, S. 27-28)

Kompetenz erscheint in Weinerts Sichtweise ähnlich wie bei Chomsky ${ }^{21}$ als latentes Konstrukt, das von den jeweils situativen Ausprägungen der Performanz unterschieden werden muss, welche allein der Beobachtung zugänglich

\footnotetext{
${ }^{18}$ IGLU - Internationale Grundschul-Lese-Untersuchung, deutscher Ableger von PIRLS Progress in International Reading Literacy Study

${ }^{19}$ DESI - Deutsch-Englisch-Schülerleistungen-International

${ }^{20}$ Oelkers und Reusser (2008, S. 26) machen darauf aufmerksam, dass dem Kern des Kompetenzbegriffs Weinerts die Konstrukte der Handlungs-, Operations- und Begriffsschemata Aeblis $(1993,1994)$ gleichgesetzt werden können.

${ }^{21}$ Chomsky (1983) unterscheidet zwischen allgemeinem Sprachvermögen (= Kompetenz) und aktueller Sprachverwendung (= Performanz). Nur im hypothetischen Konstrukt des idealen Sprechers/Hörers ist Performanz identisch mit Kompetenz.
} 
sei. Die verschiedenen Kompetenzkonzeptionen unterscheiden sich denn auch gerade in ihren Aussagen, in welchem Grad das manifeste Verhalten in der Problemlösesituation der jeweiligen Kompetenz entspricht bzw. wie scharf die Trennung zwischen Kompetenz und Performanz zu ziehen ist (vgl. Maag Merki, 2009, S. 495). Während beispielsweise Grob und Maag Merki (2001, S. 60) in Anlehnung an Chomsky und Weinert von Kompetenzen als «Performanzpotentialen» sprechen, die als solche eben keinen «deterministischen», sondern «wahrscheinlichkeitstheoretischen Charakter» aufwiesen, und «notwendige, aber nicht hinreichende Voraussetzungen für das Erzeugen besagten Bewältigungsverhaltens [...] in konkreten [Problem-]Situationen» darstellten, beziehen sich die oben genannten large scale-Leistungsvergleichsstudien, aber auch die Konzeption von Bildungsstandards (vgl. Klieme et al., 2007) auf ein Kompetenzmodell, das auf den kognitiven Fähigkeiten der Individuen beruht, «konkrete Anforderungssituationen eines bestimmten Typs zu bewältigen» (S. 72). So werden «verschiedene Kompetenzen und Teilkompetenzen [...] v. a. nach den Inhalten der interessierenden Situationen, der relevanten Aufgaben und den zur Lösung dieser Aufgaben zu bewältigenden Anforderungen definiert» (Hartig \& Klieme, 2006, S. 131). Das manifeste Handeln der Schülerinnen und Schüler wird dabei mehr oder weniger ihren Kompetenzen gleichgesetzt (vgl. Maag Merki, 2009, S. 495). Die in den Leistungstests der genannten Vergleichsstudien verwendeten Kompetenzmodelle beschränken sich sodann ganz auf kognitive Aspekte und verzichten darauf, motivational-affektive und soziale Aspekte einzubeziehen (vgl. Bos, Voss \& Goy, 2009).

Für die vorliegende Studie, die u. a. untersucht, welche schulbezogenen Verhaltensweisen des Kindes im Kontext eines unsicheren Übertritts in den Fokus der elterlichen Wert- und Kontrollregulationen geraten, wurde zur Kategorisierung des Verhaltens der Kinder, das sich auf eine breite Auswahl schulischer Anforderungssituationen bezieht, auf das Klassifikationssystem von Kompetenzen nach Erpenbeck und von Rosenstiel (2003) zurückgegriffen (vgl. Abschnitt 6.4.2.1). In diesem werden Kompetenzen als «Dispositionen selbstorganisierten Handelns» (S. XV) aufgefasst und vier Kompetenzklassen unterteilt (Erpenbeck \& von Rosenstiel, 2003, S. XVI, Hervorhebungen E.S.):

Personale Kompetenzen: [...] die Dispositionen einer Person, reflexiv selbstorganisiert zu handeln, d. h. sich selbst einzuschätzen, produktive Einstellungen, Werthaltungen, Motive und Selbstbilder zu entwickeln, eigene Begabungen, Motivationen, Leistungsvorsätze zu entfalten und sich im Rahmen der Arbeit und außerhalb kreativ zu entwickeln und zu lernen. 


\begin{abstract}
Aktivitäts- und umsetzungsorientierte Kompetenzen: [...] die Dispositionen einer Person, aktiv und gesamtheitlich selbstorganisiert zu handeln und dieses Handeln auf die Umsetzung von Absichten, Vorhaben und Plänen zu richten - entweder für sich selbst oder auch für andere und mit anderen [...]. Diese Dispositionen erfassen damit das Vermögen, die eigenen Emotionen, Motivationen, Fähigkeiten und Erfahrungen und alle anderen Kompetenzen - personale, fachlich-methodische und sozial-kommunikative - in die eigenen Willensantriebe zu integrieren und Handlungen erfolgreich zu realisieren.
\end{abstract}

Fachlich-methodische Kompetenzen: [...] die Dispositionen einer Person, bei der Lösung von sachlich-gegenständlichen Problemen geistig und physisch selbstorganisiert zu handeln, d. h. mit fachlichen und instrumentellen Kenntnissen, Fertigkeiten und Fähigkeiten kreativ Probleme zu lösen, Wissen sinnorientiert einzuordnen und zu bewerten; das schließt Dispositionen ein, Tätigkeiten, Aufgaben und Lösungen methodisch selbstorganisiert zu gestalten, sowie die Methoden selbst kreativ weiterzuentwickeln.

Sozial-kommunikative Kompetenzen: [...] die Dispositionen, kommunikativ und kooperativ selbstorganisiert zu handeln, d. h. sich mit anderen kreativ auseinanderund zusammenzusetzen, sich gruppen- und beziehungsorientiert zu verhalten und neue Pläne, Aufgaben und Ziele zu entwickeln.

Die Autoren merken an, dass diese Kompetenzklassen einen breiten Rahmen vorgeben und in ähnlicher Form immer wieder benutzt würden (z. B. Weinert, 2001, S. 28), wenn eine «grundlegende Taxonomie von Kompetenzen» (Erpenbeck \& von Rosenstiel, 2003, S. XVI, Hervorhebung im Original) angestrebt werde. Erst mit der «Zuordnung von Einzel- und Teilkompetenzen» (Erpenbeck \& von Rosenstiel, 2003, S. XVI) zu diesen vier Kategorien würden sich jeweils die Ausdifferenzierungen ergeben. Deutlich wird, dass sich nebst sozialen Kompetenzen auch emotionale, motivationale und volitionale Konstrukte wie beispielsweise Selbstkompetenzen und persönliche Wertorientierungen (Kategorie: Personale Kompetenzen) oder Persistenz (Kategorie: Aktivitäts- und umsetzungsorientierte Kompetenzen) klar zuweisen lassen, was das Klassifikationssystem für die Analyse des elterlichen Unterstützungshandelns attraktiv macht (vgl. Abschnitt 6.4.2.1 und Abschnitt 6.4.2.2).

\title{
2.2.2.2 Formen der häuslichen Unterstützung in der parental involvement-Forschung
}

Der Zweck bzw. das «Oberziel» (Esser, 2001, S. 263) des häuslichen schulbezogenen Ressourceneinsatzes der Eltern, worauf die einzelnen situativen, immer auf einen konkreten «Gegenstand» bezogenen Förderhandlungen bei ihren Kindern letztlich abzielen, besteht darin, sicherzustellen, dass das Kind ein möglichst 
hohes Maß jener Kompetenzen erwirbt, welche sie selber als primäre Bildungsziele oder als instrumentell zur Erreichung von solchen definieren und von denen sie Gewissheit oder auch nur die Vermutung haben, dass die Schule sie vorsieht, fördert und misst oder auch nur im Rahmen der «grammar of schooling»-ihrer habituellen kulturellen Handlungsmuster - erwartet (vgl. Tyack \& Tobin, 1994). In der angelsächsischen parental involvement-Forschung sind eine ganze Reihe von Formen und Facetten elterlicher häuslicher Unterstützung vorgeschlagen und hinsichtlich ihrer Leistungsförderlichkeit untersucht worden. In ihrer Synthese von neun Metaanalysen ${ }^{22}$ zum Effekt von parental involvement auf academic achievement, die in den letzten 15 Jahren in den USA, Kanada und Australien durchgeführt wurden, arbeitet Wilder (2014, S. 388-390) acht Kernkonzepte des home-based involvement heraus, die in den neun Metaanalysen im Fokus standen:

- Eltern-Kind-Kommunikation über die Schule (parent-child communication about school)

- Prüfen, ob es Hausaufgaben gibt bzw. ob und wie sie erledigt wurden (checking homework)

- Hilfestellungen bei Hausaufgaben geben (homework assistance)

- Leistungserwartungen und Bildungsaspirationen (education expectations and aspirations)

- Regeln für Schularbeiten und Freizeitaktivitäten einführen und durchsetzen, Ablenkungen ausschalten (home-supervision)

- Gemeinsame Lektüre (reading with children)

- Erziehungsstil (parenting style)

- Elterliche Überzeugungen mit Bezug auf Unterricht und Schule (parental attitudes toward education)

Im Vergleich der neueren Metaanalysen zeigt sich, dass generell eine starke positive Beziehung zwischen den beiden Konstrukten parental involvement based at home und academic achievement besteht (Wilder, 2014, S. 392). Zwar erweist sich elterliche Unterstützung z. B. in den Studien von Jeynes (2007) und namentlich in der auf Hausaufgaben bezogenen Metaanalyse von Patall et al. (2008) auf der Primarstufe als besserer Prädiktor bezüglich der Leistungsentwicklung als auf der Sekundarstufe I und zeigen sich je nach ethnischer Zugehörigkeit der Kinder und Jugendlichen bei bestimmten involvement-Komponenten signifikantere Effekte,

\footnotetext{
${ }^{22}$ Fan und Chen (2001); Jeynes (2003), Jeynes (2005), Erion (2006), Jeynes (2007), Senechal und Young (2008), Patall, Cooper und Robinson (2008), Hill und Tyson (2009), Jeynes (2012)
} 
doch erweist sich die häusliche schulbezogene Unterstützung der Eltern generell über alle untersuchten Schulstufen und ethnischen Hintergründe der Familien hinweg als positiv mit den verschiedensten Formen von Leistungsmaßen ${ }^{23}$ der Kinder und Jugendlichen verknüpft (Wilder, 2014, S. 393).

Zwischen den einzelnen Formen bestehen allerdings zum Teil deutliche Unterschiede hinsichtlich ihrer Effektstärke auf die Leistung. Ebenso zeigen sich bezüglich einzelner Formen auch Inkonsistenzen in der Befundlage über die Metaanalysen hinweg. Dies gilt besonders für die elterliche Unterstützung bei Hausaufgaben.

\subsubsection{Elterliche Unterstützung bei Hausaufgaben}

Hausaufgaben sind Aufgaben, die von den Schülerinnen und Schülern außerhalb der Unterrichtsstunden - meist zu Hause - erledigt werden sollen und von den Lehrkräften hauptsächlich mit der Intention aufgegeben werden, behandelte Inhalte zu vertiefen und zu üben, neue Informationen zu sammeln sowie aktivitäts- und umsetzungsbezogene Kompetenzen wie Selbständigkeit, Persistenz, aber auch planmäßiges Vorgehen und andere Arbeitstechniken zu fördern (vgl. Lipowsky, 2012; Nilshon, 1999, S. 13-14). Wie sich auf der Basis theoretisch fundierter, längsschnittlich und mehrebenenanalytisch angelegter Studien (u. a. Lipowsky, Rakoczy, Klieme, Reusser \& Pauli, 2004; Trautwein, 2007; Trautwein, Köller \& Baumert, 2001; Trautwein, Lüdtke, Schnyder \& Niggli, 2006) in den letzten Jahren im deutschsprachigen Raum gezeigt hat (Niggli, Trautwein, Schnyder, Lüdtke \& Neumann, 2007; Wild, E. \& Lorenz, 2010, S. 119-120), scheinen weder die - «multideterminierte» und auf der Basis von Selbstberichten kaum reliabel zu messende (Trautwein, 2008, S. 565) - Zeit, die Schülerinnen und Schüler in die Bearbeitung ihrer Hausaufgaben investieren noch der Umfang

\footnotetext{
${ }^{23}$ Bezüglich der Leistungsmaße zeigen sich in den Metaanalysen generell stärkere Beziehungen, wenn Leistung global (z. B. Notendurchschnitt im Zeugnis) und nicht domänenspezifisch (z. B. an Mathematiknoten oder -testergebnissen gemessen) erfasst wird. Dies gilt allerdings nicht für die Unterstützungskomponente «Leistungserwartungen der Eltern», hier scheint die Beziehung in der Regel stärker zu sein, wenn spezifische Noten- oder Testleistungen herangezogen werden, was für die Domänenspezifität dieses Konstrukts spricht. Sodann scheinen allgemein stärkere Assoziationen zu Lehrpersonenratings und Noten als zu Ergebnissen von standardisierten Leistungstests zu verzeichnen zu sein. Wild (2004, S. 43) weist auf das grundsätzliche Problem hin, dass in Metaanalysen, die vornehmlich auf querschnittlich angelegten Studien basierten, Leistungsmaße als Outcome-Variablen herangezogen würden, ohne dass deren Multideterminiertheit mit der Kontrolle der Effekte, die von Unterrichts- und Schulmerkmalen ausgehen, Rechnung getragen werden könne. Eine ausführliche methodische Kritik speziell der auf Hausaufgaben fokussierten meist amerikanischen Metaanalysen findet sich bei (vgl. Trautwein \& Köller, 2003).
} 
der Hausaufgaben (durchschnittliche Bearbeitungszeit in der Klasse), sondern die Häufigkeit und Regelmäßigkeit der Hausaufgabenerledigung, das Engagement bei der Bearbeitung (Anstrengung und Konzentration) sowie die prozessorientierte Einbindung von Hausaufgaben in den Unterricht (u. a. Diskussion von Lösungswegen) bedeutsam für eine günstige Leistungsentwicklung zu sein. Was die Rolle der Eltern bei Hausaufgaben betrifft, so zeigt sich in Wilders Metasynthese (2014, S. 392), dass keine positive Korrelation zwischen der elterlichen Hilfestellung bei Hausaufgaben und der Leistung der Kinder und Jugendlichen in den untersuchten Metaanalysen besteht bzw. dass elterliche Hilfestellungen in den Studien von Hill und Tyson (2009) und von Jeynes (2005) sogar negativ mit der Lernleistung assoziiert sind. Widersprüchliche Befunde zeigen sich ebenso für die Komponenten checking homework und home supervision. Damit wird das Bild bestätigt, das sich in den letzten Jahren immer wieder in (Meta-)Studien gezeigt hat. So berichtet auch Hattie (2009), bei dessen Metaanalyse parental involvement gesamthaft eine förderliche mittlere Effektstärke von $d=0.51$ ausweist, von einer moderaten mittleren Effektstärke für elterliche Hilfe bei Hausaufgaben $(d=0.38)$ und lediglich von einer geringen mittleren Effektstärke von $d=0.18$ für Strukturierungsversuche (home supervision), wozu auch das Monitoring von Hausaufgaben zählt (vgl. Hattie, 2009, S. 70). Negative Effekte seien immer dann festzustellen, wenn dem elterlichen Handeln ein «surveillance approach» (Hattie, 2009, S. 68) zugrunde liege. Es sind ähnliche Befunde, die sich in den meist auf querschnittlich angelegten Einzelstudien und auf Leistung als Zielvariable beruhenden Metaanalysen schon länger zeigen (vgl. Patall et al., 2008, S. 1092) und vermutlich damit erklärt werden können, dass es vielen Eltern, je nach eigenem Bildungshintergrund und Domäne in fachlich-inhaltlicher Hinsicht mit zunehmender Klassenstufe immer schlechter gelingen dürfte, dem Kind adäquate Hilfestellungen zu geben (vgl. Scott-Jones, 1995, S. 96-99). Ebenso dürften wie dies längsschnittlich angelegte Studien nahelegen (z. B. Pomerantz \& Eaton, 2001) - Eltern gerade dann, wenn die Leistungen des Kindes nicht ihren Erwartungen entsprechen, selber aktiv werden, und im positiven Fall einen «socratic approach» (Lepper \& Woolverton, 2002, S. 152) wählen, indem sie im Sinne eines kontingenten Scaffoldings (vgl. Wood, Bruner \& Ross, 1976; Wood \& Middleton, 1975) das Problemlösehandeln des Kindes in adaptiver Form nur bei jenen Aspekten unterstützen, die es nicht selber meistern kann (vgl. Vygotskij, 2002) und dann bemüht bleiben, nur so viele und so deutliche Hinweise zu geben wie nötig (zsf. Belland, 2014; Collins \& Kapur, 2014; Reiser \& Tabak, 2014; Reusser \& Pauli, 2015). Experimentelle Studien zeigen, dass ein solches Vorgehen von Eltern konsistent positiv mit dem nachfolgenden Meistern ähnlicher Problemlöseaufgaben durch das Kind allein assoziiert ist (zusf. Pino-Pasternak \& Whitebread, 2009). 
Gelingendes Tutoring dieser Art bedingt allerdings nicht nur, dass die Eltern über das notwenige fachliche Wissen verfügen, sondern ebenso darauf bedacht sind, neben den kognitiven Aspekten stets auch die sozioemotionale Dimension im Auge zu behalten:

[... the] best tutors are those who are concerned simultaneously with student's learning on the one hand and their motivation on the other. [...] And, when they are at their best, $[\ldots]$ they are producing both high levels of student interest and attention and extensive learning in a quite limited period. (Lepper \& Woolverton, 2002, S. 151-152, Hervorhebung im Original)

Vermutlich liegen hierin die zwei Hauptgründe, warum es zur inkonsistenten Befundlage in den Metanalysen zur elterlichen Hausaufgabenunterstützung kommt: Im negativen Fall sind Eltern bezüglich des Fachwissens überfordert und wenn sie sich aufgrund der unbefriedigenden Leistungen ihres Kindes zu einem verstärkten Engagement bei Hausaufgaben entschließen, wählen sie Erklärungsansätze und Lösungsstrategien, die nur geringe Kongruenz zu denjenigen der Lehrkräfte aufweisen. Dabei zeigen sie sich ungeduldig und enttäuscht, ob der Frustration und der Demotivation des Kindes beim gemeinsamen Arbeiten, reagieren genervt, äußern Kritik, drohen mit Strafe und erhöhen den Druck - kurz sie handeln unter Umständen in einem direktiven und zugleich wenig diskursiven Kommunikationsmodus, was wieder zu Reaktanz auf Seiten des Kindes führen dürfte. Tatsächlich berichten Eltern und Kinder immer wieder von Konflikten und Streit im Kontext von Hausaufgaben (und ungenügenden Noten) (vgl. Fuhrer, 2009, S. 266; Moroni, Dumont \& Trautwein, 2016; Neuenschwander et al., 2005, S. 60-62; Niggli et al., 2007; Ulich, 1993, S. 144; Zaugg, 2014, S. 118-119) und verschiedentlich wird darauf verwiesen, dass solcherlei einmischendes Verhalten bei Hausaufgaben gerade mit Anbruch der Pubertät, wenn Kinder und Jugendliche in gesteigertem Maß Unabhängigkeit von den Eltern und Selbstbestimmtheit suchten, besonders dysfunktional sein dürfte:

Thus, although providing guidelines for homework behavior or providing direct help with homework may be an effective form of involvement for elementary students, as students reach adolescence, it may be important that parents gradually withdraw from the homework process and shift their involvement more to support of the child's own autonomous efforts. (Patall et al., 2008, S. 1089) 


\subsubsection{Die Bedeutung elterlicher schulbezogener Erwartungen und Aspirationen und des Stils ihrer Vermittlung für die Motivation und das Engagement des Kindes}

Damit wird deutlich, dass auf Seiten der Eltern der Stil ihres schulbezogenen Handelns sowie Haltungen und Überzeugungen dem Kind und den schulbezogenen Zielen gegenüber (vgl. Abbildung 2.1), die diesem Handeln zugrunde liegen, entscheidender und einflussreicher sein dürften als das Ausmaß ihres instruktionalen Handelns im Rahmen von Hausaufgaben: «[...] how parents become involved determines in large parts the success of their involvement» (Pomerantz et al., 2007, S. 373). So erweisen sich denn auch die kindbezogenen Leistungserwartungen und Bildungsaspirationen (education expectations and aspirations) - bemerkenswerterweise im engeren Sinne kognitive Konstrukte und keine behavioralen (vgl. Abschnitt 4.2) - sowie der elterliche Unterstützungsstil in den neueren Arbeiten zum parental involvement als die bei weitem effektstärksten Konstrukte hinsichtlich der schulbezogenen Merkmale des Kindes. Jeynes (2005) schreibt dazu im Kommentar seiner Metaanalyse:

One definite pattern that emerged is that some of the most potent facets of parental involvement are some of the more subtle aspects of family support. Most notably parental expectations and style each demonstrated a strong relationship with scholastic outcomes. Thus, it was not particular actions such as attending school functions, establishing household rules, and checking student homework that yielded the statistically significant effect sizes. Rather, variables that reflected a general atmosphere of involvement produced the strongest results. Parental expectations and style may create an educationally oriented ambience, which establishes an understanding of a certain level of support and standards in the child's mind. (Jeynes, 2005, S. 262)

«Expectations» verweisen auf die kindbezogene Wahrnehmungen und Überzeugungen der Eltern und «style» verweist auf deren typische, relativ stabilen Merkmale im Umgang mit dem Kind (vgl.Hock, 2008, S. 492; Simpkins et al., 2015a, S. 617). Beide Konstrukte charakterisieren die jeweilige Beziehung zwischen den Eltern und dem Kind und sind geprägt von der dialektischen Spannung, die zwischen Erziehenden und Erzogenen grundsätzlich besteht: «Auf der einen Seite [steht] derjenige/diejenige mit Überlegenheitsmerkmalen des Alters-, Wissens- und Erfahrungsvorsprungs, [...] auf der anderen Seite derjenige/diejenige mit den Unterlegenheitsmerkmalen des Jüngerseins, des recht vorläufigen Wissens, der mangelnden Lebenserfahrung und mit einem Status minor behafteten» (Bönsch, 2006, S. 151), der/die aber ein sich entwickelndes Subjekt ist, das im Sinne des in Abschnitt 2.1 dargelegten Menschenbildes des «Resourceful-Restricted-Expecting-Evaluating-Maximizing-Man» (Esser, 1999a, 
S. 238) auf der Grundlage seiner Persönlichkeitsmerkmale und Erfahrungen seinen eigenen Interessen, Aspirationen und Zielen nachgeht und seine Akzeptanz der «angebotenen» Werte und Orientierungen nicht zuletzt davon abhängig macht, ob sie in einer seinen Bedürfnissen nach Selbstbestimmung, sozialer Anerkennung und Selbstwirksamkeit entgegenkommenden Art und Weise vermittelt werden (vgl. Ryan \& Deci, 2002; Vansteenkiste, Lens \& Deci, 2006). Was im Strukturmodell (vgl. Abbildung 2.1) idealisierend als symmetrische Beziehung dargestellt ist, ist in der Erziehungswirklichkeit wegen der genannten «Vorsprünge» (Bönsch, 2006, S. 153) der älteren Generation immer grundlegend asymmetrisch. Doch müssen sich die Eltern in dieser Sichtweise, immer wieder der Intentionen und des Sinnzusammenhangs des Kindes in den jeweiligen Situationen bewusst werden (vgl. Mollenhauer, 1972, S. 126-133), um ihre erzieherischen Ziele nachhaltig zu erreichen, und daran anknüpfen - was Dialog, «Ko-Konstruktion» (Reusser \& Pauli, 2015) oder «diskursive Auseinandersetzung»(Oelkers, 2009, S. 255), aber auch Infragestellung eigener Positionen und das Aushalten und Klären von Missverständnissen und Mehrdeutigkeiten beinhaltet. In der Spannung zwischen «'Freiheit und Bindung', 'Bewahren und Verändern', 'Planung und Offenheit', 'Führen oder Wachsenlassen', 'Gewährenlassen und Fordern', 'Vertrautheit und Distanz', 'Individualität und Soziabilität'» (Bönsch, 2006, S. 163) ist es gemäß den Postulaten der empirisch orientierten Forschung um Erziehungsstile (zusf. Baumrind, 1989; Hock, 2008; Tausch \& Tausch, 1998) wichtig, auf häusliche Praktiken zu setzen, die von gegenseitigem Abstimmen, Aushandeln und Erklären geprägt sind und bei denen weder der «Einsatz von elterlicher Autorität» noch die «Berücksichtigung von kindlichen Bedürfnissen» einseitig überhandnimmt (Hurrelmann, 2006, S. 162).

Nach einer kurzen ersten Erörterung der Bedeutung von elterlichen schulbezogenen Erwartungen und Aspirationen (vgl. auch Kapitel 5) wird im Folgenden die «Kommunikations- [...] und Beziehungskultur» (Reusser, 2008, S. 228) zwischen den Eltern und Kindern noch genauer in den Blick genommen und Kernkomponenten eines (motivations-)förderlichen elterlichen Handlungsstils herausgearbeitet.

Als klar einflussreichste Komponente elterlicher häuslicher Unterstützung erweist sich in den Metaanalysen die Kategorie elterliche Leistungserwartungen und Bildungsaspirationen (Fan \& Chen, 2001; Hill \& Tyson, 2009; Jeynes, 2005, 2007). Hattie (2009) berichtet für die beiden Konstrukte zusammengenommen eine sehr hohe mittlere Effektstärke auf Schulleistungen von $\mathrm{d}=0.80$. Mit dem Konstrukt Erwartungen werden prinzipiell «Annahmen oder Hypothesen» (Greitemeyer, Fischer \& Frey, 2006, S. 336) «about events or behaviors that will occur 
or that will be revealed in the future» (Hoorens, 2012, S. 142) bezeichnet. Beziehen sich behaviorale Erwartungen auf das Selbst, spricht man von intrapersonellen Erwartungen (vgl. Abschnitt 5.3). Handelt es sich wie im vorliegenden Fall um Annahmen über das Verhalten oder die Kompetenzen anderer, ist von interpersonellen Erwartungen die Rede (vgl. Dresel \& Lämmle, 2017, S. 124). Erwartungen können einen deskriptiven Charakter aufweisen, wenn sie zum Ausdruck bringen, was gemäß der Ansicht des Individuums mit einer bestimmten Wahrscheinlichkeit eintreffen wird, oder sie können normativer Natur sein, wenn sie mit einer präskriptiven Konnotation zum Ausdruck bringen, was «for logical, social, or moral reasons» (Hoorens, 2012, S. 142) passieren sollte ${ }^{24}$. Aspirationen, die ebenfalls intra- und interpersoneller Form sein können, bezeichnen demgegenüber die Ziele, die mit der Hoffnung verbunden sind, dass man sie selber erreicht oder dass andere sie erreichen (vgl. Goldenberg et al., 2001, S. 548).

Da Erwartungen und Aspirationen kognitive Konstrukte und nicht eigentliche Verhaltens- oder Handlungsweisen bezeichnen, sind sie in einigen Metaanalysen mit der Komponente Eltern-Kind-Kommunikation über Schule vermengt (vgl. Abschnitt 2.2.2.2). So fassen z. B. Hill und Tysen die beiden Facetten des parental involvements unter der Kategorie der academic socialization zusammen:

\begin{abstract}
Academic socialization includes parents' communication of their expectations for achievement and value for education, fostering educational and occupational aspirations in their adolescents, discussing learning strategies with children, and making preparations and plans for the future, including linking material discussed in school with students' interests and goals. (Hill \& Tyson, 2009, S. 754)
\end{abstract}

Die Definition betont die Bedeutung der Externalisierung elterlicher Werte, Aspirationen und Erwartungen mit nahem Bezug zu den Inhalten, Fragen und Ereignissen, mit denen das Kind in Unterricht und Schule konfrontiert ist. Ebenso wird allein schon im Terminus «socialisation» deutlich, dass diese die schulische Realität des Kindes interpretierenden und gewichtenden kommunikativen Ereignisse zwischen Eltern und Kind zwar auch, aber nicht ausschließlich in genuin schulbezogenen Aktivitäten wie Hausaufgaben oder Prüfungsvorbereitungen vorkommen können, sondern in jeglichen gemeinsamen häuslichen Aktivitäten.

\footnotetext{
${ }^{24}$ Normative Erwartungen sind denn auch eng verbunden mit persönlichen Normen bzw. Werten (vgl. Rokeach, 1973; Rokeach, 1979). Wenn in der vorliegenden Arbeit von «Erwartungen» gesprochen wird, so sind in der Regel intra- oder interpersonelle deskriptive, mit Kontroll-Überzeugungen verbundene zukunftsgerichtete Annahmen gemeint. Wenn der normative Aspekt im Vordergrund steht, wird von «Wert-Überzeugungen» oder «WertZuschreibungen» bzw. vom subjective task value gesprochen (vgl. Abschnitt 5.5.1).
} 
Elterliche Haltungen und Überzeugungen können explizit in Gesprächen thematisiert (vgl. Kapitel 5) oder aber subtiler über elterliche Modellierungsleistungen (vgl. Bandura, 1992) sowie über non-verbale Reaktionen auf ein bestimmtes Verhalten des Kindes im Sinne des Operanten Konditionierens (vgl. Skinner, B. F., 1989) vermittelt werden. Während Kinder im ersteren Fall beobachten und erschließen, was ihren Eltern offenbar wichtig und teuer ist, weil diese Zeit und andere Ressourcen darauf verwenden, nehmen sie dies im letzteren Fall durch die für sie als angenehm oder unangenehm empfundenen Konsequenzen unmittelbar wahr und passen ihr Denken und Verhalten entsprechend an (vgl. Abschnitt 5.6.2).

Damit ist auch angesprochen, dass sich bezüglich der Effekte der Blick nicht primär auf die Leistungsmaße, sondern auf motivationale Orientierungen und das Engagement des Kindes richten sollte (vgl. Patall et al., 2008, S. 1093). Eine Forderung, die in der Involvement-Forschung ab den 80er-Jahren des letzten Jahrhunderts unter dem Eindruck der Selbstbestimmungstheorie von Deci und Ryan (1993) durch die Arbeitsgruppe um Wendy S. Grolnick (z. B. Grolnick, 2003; Grolnick et al., 1991; Grolnick \& Slowiaczek, 1994) sowie im Anschluss an die Erwartungs-Wert-Theorie der Lern- und Leistungsmotivation durch Jacquelynne und ihre Arbeitsgruppe (z. B. Eccles \& Harold, 1996; Frome \& Eccles, 1998) erhoben und mit entsprechenden theoretischen Modellen und empirischer Forschung untermauert wurde (vgl. Abschnitt 4.2). Kathleen Hoover-Dempsey und ihre Mitarbeitenden (2001), die die Forderung unter dem Eindruck der unbefriedigenden Befundlage zum parental involvement in homework ebenfalls aufnahmen, schreiben:

Ultimately, however, a solitary emphasis on student achievement is unfortunate. Parents' homework involvement behaviors are more logically related to proximal student outcomes (e.g., attitudes about homework, perceptions of personal competence) than to student performance on summary assessments of achievement. The power of these proximal variables rests in the reality that student achievement ultimately depends not only on parents' behaviors, but on variables that are often (and increasingly, across the course of development) outside of parents' control (e.g., classroom instruction, student decisions to use skills, knowledge and related strategies in learning tasks). Thus, the most critical outcomes associated with parental involvement in homework may be found in the attitudes, ideas, and behaviors enacted by students in the course of school learning [...]. (Hoover-Dempsey et al., 2001, S. 204)

Der Ansatz Grolnicks (vgl. Grolnick \& Ryan, 1989) zur Klassifikation von Qualitätsdimensionen elterlichen Hausaufgabenverhaltens hat über die Jahre eine breite Rezeption, u. a. auch in deutschsprachigen Ländern, gefunden (u. a. Buff, Reusser \& Dinkelmann, 2017; Exeler \& Wild, 2003; Lorenz \& Wild, 2007; 
Niggli et al., 2007; Wild, E., Rammert \& Siegmund, 2006; Wild, E. et al., 2005). Die Dimensionen basieren auf den Postulaten der Selbstbestimmungstheorie und entsprechender u. a. im Unterricht gewonnener Befunde (Ryan \& Deci, 2000b, 2002; Vansteenkiste, Ryan \& Deci, 2008), wonach jene Situationen mit positiver Lernmotivation, günstigem Lernhandeln und Wohlbefinden einhergehen, welche vom Individuum als in Einklang mit seinen Grundbedürfnissen nach Autonomie, Kompetenz und sozialer Eingebundenheit erlebt werden. Die Dimensionen elterlichen Unterstützungshandelns bei Hausaufgaben werden vor diesem Hintergrund danach beurteilt, inwieweit sie den genannten Grundbedürfnissen des Kindes entgegenkommen. Ellen A. Skinner und Kolleg*innen (2009) haben in einer Übersichtsarbeit die in der Zwischenzeit auf dieser theoretischen Grundlage ebenso wie die ähnlich konzipierten, aber in den letzten Jahrzehnten im Kontext der Erziehungsstilforschung (z. B. Baumrind, 1967, 1991; Weiss \& Schwarz, 1996) vorgeschlagenen Qualitätsmerkmale elterlichen Verhaltens in einer Übersicht zusammengetragen und zu sechs Kerndimensionen verdichtet (vgl. Tabelle 2.1). In Einklang mit der bisherigen - konsistenten - Befundlage in beiden Forschungslinien korrelierten die drei Merkmale Wärme, Struktur, Autonomieunterstützung in zwei von den Autorinnen durchgeführten Studien ${ }^{25}$ positiv mit motivationalen Aspekten (perceived academic competence, self-worth) mit Engagement (commitment to school, mastery) und negativ mit problematischem Verhalten (substance use, problem behaviors). Ebenso erwartungsgemäß verhielt es sich bezüglich der genannten Zielgrößen genau umgekehrt mit den drei Dimensionen Ablehnung, Chaos und Zwang (vgl. Skinner, E. A. et al., 2009, S. 221) ${ }^{26}$.

\footnotetext{
${ }^{25}$ Die Studien werden von Skinner, E. A. et al. (2009) im gleichen Artikel erörtert wie die in Tabelle 2.1 dargestellte Übersicht über die Kernkonstrukte.

${ }^{26}$ Die Autorinnen halten fest, dass die sechs Qualitätsmerkmale im Rahmen von «traditional bipolar models» (Skinner, E. A. et al., 2009, S. 196) jeweils als entgegengesetzte Pole auf den drei Dimensionen elterliche Wärme und Anteilnahme (Wärme vs. Zurückweisung), elterliche Strukturgebung (Struktur vs. Chaos) sowie elterliche Autonomieunterstützung (Autonomieförderung vs. Zwang) konzipiert würden. Strukturanalysen auf der Basis der Daten zweier unabhängiger Erhebungen bei Müttern und Vätern sowie bei Jugendlichen belegten, dass diese sechs Merkmale allerdings besser als je eigenständige, unterscheidbare Dimensionen konzipiert werden sollten, insofern als sie auf diese Weise eine signifikant bessere Passung mit der den Eltern- und Jugendlichen-Einschätzungen unterliegenden Dimensionalität aufwiesen, als wenn sie jeweils als gegenüberliegende Pole konzipiert würden. Die Korrelationen, die zwischen den positiven Merkmalen Wärme, Struktur und Autonomieunterstützung sowie zwischen den negativen Merkmalen Zurückweisung, Chaos und Zwang in den beiden Teilstudien auftraten, sprächen ferner für die Aggregation dieser Skalen, wie dies in der Vergangenheit in der parenting-Forschung z. B. mit den Erziehungsstilen autoritativ oder autoritär vorgenommen worden sei. Ferner deute der Umstand, dass die sechs Merkmale unterschieden werden können, auf die Validität von Typologien elterlichen Verhaltens hin, die Baumrind (1991)
} 
Unterstützungsstile sind Aggregationen aus diesen Dimensionen. In zwei Studien mit Eltern und ihren Kindern - einmal zum Thema Mathematikhausaufgaben mit 300 Drittklässlern (Wild, E. \& Remy, 2002) und eine weitere mit dem Fokus auf Chemiehausaufgaben mit 215 Siebtklässlern im Gymnasium (Exeler \& Wild, 2003) - zeigte die Forschungsgruppe um Elke Wild, dass zwar nur ein kleinerTeil der Eltern mit Blick auf die Postulate der Selbstbestimmungstheorie klar dysfunktionale Unterstützungsstile praktiziert (vernachlässigend oder überbehütend), aber ebenso wenige einen optimalen Unterstützungsstil an den Tag legen, der von ihnen als «strukturierende Begleitung» (autonomieunterstützend, strukturierend, emotional-zugewandt und prozessorientiert) bezeichnet wird. Die große Mehrheit (über $70 \%$ ) der Eltern und Kinder berichten sowohl vom Einsatz förderlicher wie auch hinderlicher Formen - u. a. geringe Prozessorientierung im Sinne des oben beschriebenen kontingenten Scaffoldings bis hin zu einschränkendem, überkontrollierendem Vorgehen der Eltern. Wild und Remy (2002, S. 285) glauben, dass mit erhöhtem Leistungsdruck vor dem Übertritt in die Sekundarstufe I «mit einer Verstärkung kontrollierender und einer Verringerung emotional- und autonomieunterstützender Reaktionen» der Eltern zu rechnen sei (vgl. auch Grolnick, Price, Beiswenger \& Sauck, 2007). Sie stützen ihre Vermutung darauf, dass bei den Drittklässlern, die rund ein Jahr vor dem Übertritt stehen, bereits ein Zusammenhang zwischen der Leistungsfähigkeit und dem Ausmaß der von ihnen berichteten $\left(\mathrm{r}=-.37^{*}\right)$ gegenüber der von den Eltern $\left(\mathrm{r}=-.23^{*}\right)$ berichteten intrusiven Kontrolle (Zwang) beobachtbar sei. In ihrer längsschnittlich angelegten Studie zum Hausaufgabenverhalten von Eltern im Fach Chemie bestätigten sich sodann bei Kontrolle der Leistungsfähigkeit und der sozialen Herkunft die gemäß den Postulaten der Selbstbestimmungstheorie erwarteten Zusammenhangsmuster zwischen elterlichen Verhaltensmerkmalen bzw. Einstellungen und motivationalen Merkmalen der Kinder: Schülerinnen und Schüler, die sich von ihren Eltern bei Hausaufgaben emotional, autonomieförderlich und prozessorientiert unterstützt fühlten, berichteten zu den beiden nächsten Messzeitpunkten über ein höheres individuelles Interesse und höhere identifizierte Motivation, Aufgabenorientierung und Anstrengung im Fach Chemie. Nahmen die Kinder Leistungsdruck, überhöhte Ansprüche und ein produktorientierter Umgang mit Hausaufgaben in Chemie wahr, ging dies nachfolgend mit höherer externaler Motivation und einer ausgeprägteren Abneigung gegenüber dem Fach einher (vgl. Exeler \& Wild, 2003).

oder Weiss und Schwarz (1996) vorgebracht hätten: «If autonomy support (supportive control) can be distinguished from structure (assertive control) and coercion (directive control), then different combinations of these features can be used to characterize different types of parenting. [...] Of course, typologies could demarcate parenting styles even more clearly if they incorporated all six dimensions» (Skinner, E. A. et al., 2009, S. 196). 
Niggli et al. (2007) können in einer längsschnittlich und mehrebenenanalytisch angelegten Studie mit 1'444 Achtklässlern im Fach Französisch als Fremdsprache zeigen, dass Schülerinnen und Schüler aus Bildungsgängen mit tieferem Anforderungsgrad sowie leistungsschwächere Jugendliche (Halbjahresnoten) über signifikant mehr problematische Unterstützungsformen (intrusive Kontrolle und Streit) berichten. Längsschnittlich zeigte sich ferner, dass schlechte Französischnoten des Kindes mit zunehmender Einmischung durch die Eltern einherging und diese wiederum mit einer Verschlechterung der Testleistungen verknüpft war. In Einklang mit den Postulaten der Selbstbestimmungstheorie vermuten die Autorinnen und Autoren, die oben bereits skizzierte negative Dynamik zwischen unbefriedigenden Leistungen, problematischem elterlichem Unterstützungsverhalten und Reaktanz der Jugendlichen, die ihre Bedürfnisse nach Autonomieund Kompetenzerleben durch die unerbetene Hilfe beeinträchtigt sehen dürften. Bemerkenswerterweise war bei Kontrolle von Bildungsgang und Schulleistung ein deutlicher Geschlechtseffekt zu verzeichnen: Jungen berichteten über signifikant mehr intrusive Kontrolle und über Streit bei Hausaufgaben und wiesen auch längsschnittlich ein ungünstigeres Verlaufsmuster auf als die Mädchen. In einer im Rahmen der TRANSITION-Studie durchgeführten längsschnittlichen Untersuchung (3 Erhebungszeitpunkte zwischen der 1. und 6. Klasse, 4 Erhebungszeitpunkte zwischen der 6 und 7. Klasse) zeigen Dinkelmann und Buff (2016) auf der Basis der Daten von 457 Kindern und ihren Eltern mit Hilfe von Strukturgleichungsmodellen, dass die von Eltern berichtete intrusive Kontrolle ${ }^{27}$ erwartungsgemäß einen negativen Effekt auf mathematikbezogene Kompetenzüberzeugungen der Kinder und - vermittelt über dieselben - auf die Leistungen in Mathematik ausübte. Elterliche selbsteingeschätzte Wärme beim Unterstützen (warmth, vgl. Tabelle 2.1) erwies sich als positiver Prädiktor für die Kompetenzüberzeugungen sowie den intrinsischen Wert, den die Kinder der Mathematik zuschrieben, allerdings nur, wenn die Kinder das Unterstützungsverhalten der Eltern selber als emotional zugewandt empfanden. Ebenfalls vermittelt über die kindperzipierte Wärme sowie über dessen Kompetenzüberzeugungen erwies sich das selbsteingeschätzte warm-emotionale Verhalten der Eltern positiv prädiktiv bezüglich des Leistungsverlaufs des Kindes in Mathematik.

In der Zwischenzeit kann auf der Grundlage einer großen Zahl von Studien vor allem als gesichert gelten, dass autonomieunterstützendes Handeln der Eltern (autonomy support) mit positiven Formen bzw. günstigen Verläufen bezüglich der Motivation (Wert- und Kontrollüberzeugungen, Selbstwert), des Lernengagements und der Selbstregulation sowie bezüglich der Leistung des Kindes assoziiert ist. Die Verhaltensform, die Skinner et al. (2009) als Zwang (coercion) bezeichnen und durch die Facetten unerbetene, intrusive Hilfestellungen und

\footnotetext{
${ }^{27}$ Bei Kontrolle der vorangehenden Leistung, der Lernmotivation, der Intelligenz und des Geschlechts (vgl. Dinkelmann \& Buff, 2016).
} 
Überwachung sowie unflexible durch Strafe oder Strafandrohungen unterstrichene Regeldurchsetzung gekennzeichnet sehen ${ }^{28}$ (vgl. Tabelle 2.1) - ist dahingegen mit ungünstigen motivationalen und behavioralen Aspekten verbunden: «[...] when children perceive their parents as controlling, they are more likely to experience extrinsic motivation for learning, which is related to more negative motivational and academic outcomes. [...] the results are consistent across [...] a wide range of ages and indicators of school motivation [...]» (Grolnick, Friendly \& Bellas, 2009, S. 284). In ihrem Forschungsüberblick betonen Grolnick et al. (2009, S. 284) sodann, dass es für die beiden Unterstützungsformen zumindest starke Hinweise für den oben dargestellten reziproken Einfluss zwischen Kindund Eltern-Verhalten gibt: Eltern reagieren auf Kinder, die sie als kompetent wahrnehmen, autonomieförderlich und im umgekehrten Fall stärker intrusiv und überwachend.

Abschließend soll nun noch der Kerndimension Strukturgebung größeres Augenmerk geschenkt werden, die für die vorliegende Studie über elterliche Motivierungsversuche von besonderem Interesse sein dürfte, insofern als sie das «richtige» Informieren des Kindes über wünschbares und erwartetes Verhalten thematisiert.

Elterliche Strukturgebung (structure, vgl. Tabelle 2.1) - konzeptionell und empirisch in der Vergangenheit laut Grolnick und Pomerantz (2009) oft mit Zwang bzw. intrusive control vermischt und entsprechend inkonsistente Befunde verursachend - wird von ihnen in Einklang mit Skinner et al. (2009) «as a relatively orthogonal dimension»(S. 67) zu elterlichem Zwang als elterliche «guidance» (S. 65) konzipiert:

When parents are structuring, they highlight the relations between actions and outcomes through clear and consistent guidelines, expectations, and rules for children; they also provide children with predictable consequences for and clear feedback about their actions. (Grolnick \& Pomerantz, 2009, S. 67)

Farkas und Grolnick (2010) zeigen auf der Basis einer kombinierten Interviewund Fragebogenstudie mit 75 Siebt- und Achtklässlern und ihren Eltern ${ }^{29}$, dass Strukturgebung unabhängig von Autonomieunterstützung vs. Zwang ist, einen

\footnotetext{
${ }^{28}$ Vgl. auch Grolnick und Pomerantz (2009, S. 167): «parents’ pressure, intrusiveness, and dominance in relation to children's feelings and thoughts as well as their behavior».

${ }^{29}$ Die Autorinnen sprechen wegen der geringen Anzahl an Proband*innen und der Limitierungen, die sich daraus für die Faktoranalysen und andere statistische Prüfverfahren ergaben, von einer «Exploration» und wollen die Ergebnisse bis zur Prüfung an einem größeren Sample als «Hypothesen» verstanden wissen (Farkas \& Grolnick, 2010, S. 277).
} 
Tabelle 2.1 Sechs Kerndimensionen elterlichen Verhaltens, Definitionen und ähnliche Konstrukte (Skinner, E. A. et al., 2009, S. 186, Übersetzung und leichte Adaption E.S.)

\begin{tabular}{|c|c|c|}
\hline Dimension & Definition & ähnliche Konstrukte \\
\hline Wärme (warmth) & $\begin{array}{l}\text { Ausdruck von Liebe, Zuneigung, } \\
\text { Fürsorglichkeit und Freude. Anerkennung } \\
\text { und emotionale Verfügbarkeit. } \\
\text { (Expression of love, affection, caring, and } \\
\text { enjoyment. Appreciation, emotional } \\
\text { availability.) }\end{array}$ & $\begin{array}{l}\text { approving, acceptance, love, } \\
\text { support, supportive control, } \\
\text { positive involvement, closeness, } \\
\text { connection, child-centered }\end{array}$ \\
\hline Ablehnung (rejection) & $\begin{array}{l}\text { Aktive Abneigung, Aversion und } \\
\text { Feindseligkeit. Barsch, überreagierend, } \\
\text { auffahrend, kritisch, ablehnend. } \\
\text { (Active dislike, aversion, and hostility. } \\
\text { Harsh, over-reactive, irritable, critical } \\
\text { disapproving.) }\end{array}$ & $\begin{array}{l}\text { deprecation, hostility, harsh, } \\
\text { disapproval, negativity, cold, } \\
\text { derogation, critical, over-reactivity, } \\
\text { aversion, irritability, dislike, } \\
\text { irritable explosive discipline }\end{array}$ \\
\hline $\begin{array}{l}\text { Struktur-gebung } \\
\text { (structure) }\end{array}$ & $\begin{array}{l}\text { Zurverfügungstellung von Informationen } \\
\text { über mögliche Mittel zur Zielerreichung. } \\
\text { Berechenbar, konsistent. Klare } \\
\text { Erwartungen, verbindliche (altersgemäße) } \\
\text { Verhaltensanforderungen. } \\
\text { (Provision of information about pathways } \\
\text { to reach desired outcomes. Predicable, } \\
\text { consistent. Clear expectations, firm } \\
\text { maturity demands.) } \\
\text { «[E]xtent to which parents provide clear } \\
\text { and consistent guidelines, expectations, } \\
\text { and rules for child behavior.» (Grolnick \& } \\
\text { Ryan, 1989, S. 144) }\end{array}$ & $\begin{array}{l}\text { demandingness, firm control, } \\
\text { behavioral control, contingent } \\
\text { responsiveness, behavior } \\
\text { contingency, directive, strictness, } \\
\text { supervision, organization, } \\
\text { regulation, rule-setting, regularity } \\
\text { of routine, household organization, } \\
\text { assertive control }\end{array}$ \\
\hline Chaos (chaos) & $\begin{array}{l}\text { Sich in den Prozess der Zielerreichung } \\
\text { einmischen oder Verschleiern möglicher } \\
\text { Mittel zur Erreichung von Zielen. } \\
\text { Unstetig, widersprüchlich, erratisch, } \\
\text { unberechenbar, willkürlich oder } \\
\text { unzuverlässig. } \\
\text { (Interferes with or obscures the pathways } \\
\text { from means to ends. Noncontingent, } \\
\text { inconsistent, erratic, unpredictable, } \\
\text { arbitrary, or undependable.) }\end{array}$ & $\begin{array}{l}\text { Permissiveness, non-directive, lax } \\
\text { control, laxness, unpredictable, } \\
\text { undependable, non-contingent, } \\
\text { erratic, casual, under-controlled, } \\
\text { laissez faire, inconsistent discipline }\end{array}$ \\
\hline
\end{tabular}


Tabelle 2.1 (Fortsetzung)

\begin{tabular}{l|l|l}
\hline Dimension & Definition & ähnliche Konstrukte \\
\hline $\begin{array}{l}\text { Autonomie-unterstützung } \\
\text { (autonomy support) }\end{array}$ & $\begin{array}{l}\text { Erlaubnis zur Rede- und Handlungs- bzw. } \\
\text { Entscheidungsfreiheit. Das Kind } \\
\text { ermuntern, seine eigenen Präferenzen und } \\
\text { Meinungen wahrzunehmen, zu } \\
\text { akzeptieren und wertzuschätzen. } \\
\text { (Allow freedom of expression and action. } \\
\text { Encourage child to attend to, accept, and } \\
\text { ralue genuine preferences and opinions.) } \\
\text { permissiveness, non-directive, } \\
\text { autonomy-granting, supportive } \\
\text { control }\end{array}$ \\
& $\begin{array}{l}\text { «[D]egree to which parents value and use } \\
\text { techniques which encourage independent } \\
\text { problem-solving, choice, and } \\
\text { participation in decisions.» (Grolnick \& } \\
\text { Ryan, 1989, S. 144) }\end{array}$ & \\
\hline Zwang(coercion) & $\begin{array}{l}\text { Einschränkender, überkontrollierender, } \\
\text { aufdringlicher, autokratischer Stil. Strikter } \\
\text { Gehorsam wird verlangt. } \\
\text { (Restrictive overcontrolling intrusive } \\
\text { autocratic style. Strict obedience is } \\
\text { demanded.) } \\
\text { «[E]xternally dictating outcomes, and } \\
\text { motivating [...] through punitive } \\
\text { disciplinary techniques, pressure, or } \\
\text { controlling rewards.» (Grolnick \& Ryan, } \\
1989, \text { S. 144) }\end{array}$ & $\begin{array}{l}\text { arbitrary control, demandingness, } \\
\text { restrictiveness, autocratic, } \\
\text { psychological control, } \\
\text { controllingness, inflexible rigid } \\
\text { discipline, intrusive support, strict } \\
\text { control, over-restrictive, } \\
\text { over-controlling, power assertion, } \\
\text { intrusiveness, intrusive control }\end{array}$ \\
\hline
\end{tabular}

eigenständigen Beitrag zur Vorhersage der Motivation und Lernleistung leistet sowie aus mehreren Facetten besteht, die in unterschiedlichem Maß mit verschiedenen motivationalen und behavioralen Aspekten beim Kind assoziiert sind (S. 268-269):

a) durch Kommunizieren klarer und beständiger Regeln, Orientierungshilfen und Erwartungen (clear and consistent rules, guidelines und expectancies),

b) durch klares und konsistentes Aufklären über Handlungsfolgen und Konsequenzen (predictability),

c) durch aufgabenbezogenes, konstruktives Feedback darüber, inwiefern das Kind die Erwartungen erfüllt (task-focused information feedback),

d) durch das Schaffen von Angeboten, die es dem Kind ermöglichen, die Erwartungen und Ziele auch zu erfüllen (z. B. genug Zeit für die Hausaufgabenerledigung sowie eine adaptive Fremdregulation, wenn die Erwartung besteht, dass das Kind die Hausaufgaben vollständig erledigt) (provision of opportunities to meet expectations), 
e) durch Aufklären über die Gründe für Regeln und Erwartungen (provision of rationales for rules and expectations),

f) durch aktives Annehmen der Führungsrolle in der Familie durch Expertise beim gemeinsamen Treffen von Entscheidungen (authority).

Überblickt man die Liste, so offenbaren sich eine Reihe von Qualitätsmerkmalen der Kommunikation von unterrichts-, lern- und laufbahnbezogenen Werten, Zielen, Aspirationen und Erwartungen, bei denen augenscheinlich die assertive Funktion des Informierens und (Er-)Klärens ${ }^{30}$ im Vordergrund steht: Die Eltern als «interpreters of reality» (Jacobs \& Eccles, 2000, S. 426) deklarieren, klären auf, deuten, melden zurück, argumentieren, schlagen vor, gewichten, beziehen Positionen - immer mit der Absicht, die Aufmerksamkeit, die Rezeption, Verständlichkeit und Einsicht aufseiten des Kindes zu erhöhen. Dass dies nicht primär unidirektional im Sinne von Zwang (coersion), sondern dialogisch-partizipativ gedacht ist, wird in der Liste insbesondere bei c), d) und e) deutlich. Ebenso deutlich wird die Abgrenzung von Chaos (vgl. Tabelle 2.1) (vgl. Grolnick \& Pomerantz, 2009, S. 67) mit Blick auf den Inhalt dessen, was mitgeteilt wird (Erwartungen und Bedeutsamkeiten), und ebenso die Art, wie es kommuniziert wird (stringent, berechenbar, klar) (vgl. Abschnitt 5.7).

\subsection{Elterliche schulbezogene Unterstützung - Kommentierung der Erkenntnisse vor dem Hintergrund der vorliegenden Studie}

Elterliches schulbezogenes Unterstützungshandeln wird in der vorliegenden Studie auf der Grundlage der Postulate der Theorie der rationalen Wahl Essers als «ein an den Zielen und Bedingungen des Schulsystems orientierter Ressourceneinsatz der Eltern» bezeichnet, «der darauf gerichtet ist, die von ihnen als bedeutsam beurteilten Kompetenzen und Laufbahnschritte beim Kind zu fördern und/oder das Denken und Handeln der schulischen Akteure im Interesse des Kindes zu optimieren» (vgl. Abschnitt 2.2). Mit Blick auf das Erkenntnisinteresse der vorliegenden Studie, elterliches motivationsbezogenes Handeln bei einem unsicheren Übertritt des Kindes zu beschreiben, zu kategorisieren sowie zu beurteilen, beruht die Definition auf der Intentionalität des elterlichen Tuns

\footnotetext{
${ }^{30}$ Searle (1979, S. 12): «The point or purpose of the assertive class [of speech acts] is to commit the speaker (in varying degrees) to something's being the case, to the truth of the expressed position. All of the members of the assertive class are assessable on the dimension of assessment which includes true and false.»
} 
und grenzt «Unterstützungshandeln» von Bildungsleistungen der Eltern ab, die von ihnen laut Bourdieu (1984, 1998) auf der Grundlage ihres akkumulierten kulturellen Kapitals und ihres Habitus im Rahmen sozialisatorischer Alltagsprozesse weitgehend beiläufig und unbewusst erbracht werden (vgl. Bourdieu, 1983, S. 187) (vgl. hierzu Abschnitt 4.1.2). Mit dem in der Definition hervorgehobenen Schulbezug des elterlichen Handelns, wird dieses sodann auch vom übrigen erzieherischen Handeln der Eltern abgegrenzt, wenngleich, wie im letzten Kapitel erläutert, ähnliche Handlungsqualitäten wie sie von der Erziehungsstilforschung in den letzten Jahrzehnten herausgearbeitet wurden, darüber entscheiden dürften, wie erfolgreich es Eltern gelingt, die schulische Entwicklung ihres Kindes zu beeinflussen. In der Forschung um parental involvement hat sich in den letzten Jahren nach langjähriger Konzentration auf Leistungsmaße und einer widersprüchlichen Befundlage besonders bezüglich der elterlichen Hausaufgabenbegleitung (vgl. Abschnitt 2.2.2.3) die Erkenntnis durchgesetzt, dass Eltern generell primär einen Einfluss auf fachübergreifende Kompetenzen im personalen, motivational-volitionalen sowie im sozial-kommunikativen Bereich ausüben und folglich (zusätzlich) «proximalere» Variablen der Motivation und des Lernengagements des Kindes in den Blick genommen werden sollten. In Einklang mit den Postulaten der Selbstbestimmungstheorie (vgl. Ryan \& Deci, 2000b; Ryan \& Deci, 2002; Vansteenkiste et al., 2008) prädiktieren auf dieser Grundlage jene elterlichen Verhaltensweisen positive Formen der Lernmotivation (u. a. produktive Wert- und Kontrollüberzeugungen), günstige Merkmale des Lernhandelns und des Wohlbefindens, die den Bedürfnissen des Kindes nach Autonomie, Kompetenzerleben und sozialer Eingebundenheit entgegenkommen (vgl. Skinner, E. A. et al., 2009, S. 190): Elterliche Wärme (warmth) erweist sich als speziell förderlich für das Zugehörigkeitsgefühl (bzw. für die Bindung zwischen Kind und Eltern, vgl. Kapitel 4) von Kindern, Ablehnung (rejection) hat einen gegenteiligen Effekt. Elterliche Strukturierung (structure) erweist sich besonders wichtig für Kontrollüberzeugungen des Kindes, während Chaos (chaos) einen gegenteiligen Effekt ausübt. Elterliche Autonomieunterstützung (autonomy support) bildet sodann die Grundlage dafür, dass sich Kinder als selbstbestimmt in ihren Entscheiden und Handlungen erleben, wohingegen Zwang (coercion) diesbezüglich einen unterminierenden Effekt zeitigt.

Für die vorliegende Studie, die die elterlichen Bemühungen, mittels expliziten Bedeutsamkeitszuschreibungen und evaluativen Feedbacks positiv auf die Kontroll- und Wert-Überzeugungen ihrer Kinder einzuwirken, nicht nur beschreiben und klassifizieren, sondern auch bezüglich ihrer Förderlichkeit beurteilen will, sind die sechs Kerndimensionen elterlichen Verhaltens aber insofern von besonderer Bedeutung, als sie die Einschätzung davon erlauben, wie die wert- 
und kontrollbezogenen Botschaften der Elternteile von den Kindern wohl rezipiert werden dürften. Ob die (externalen) elterlichen Appelle das Potential haben, vom Kind angenommen und handlungswirksam internalisiert $\mathrm{zu}$ werden, dürfte nicht nur von der Plausibilität der transportierten Botschaft der Wert- oder Kontrollregulation («Es ist bedeutsam, dass du..., weil du...» bzw. «Du hast ... gut/schlecht im Griff, weil du...») abhängen, sondern - so postuliert es die Selbstbestimmungstheorie und belegen es zahlreiche Befunde (zsf. Grolnick \& Ryan, 1989; Pomerantz \& Grolnick, 2017; Wigfield, Eccles, et al., 2015, S. 24-25) auch von der Art und Weise des Appellierens sowie von der Wahrnehmung der Beziehung zum appellierenden Elternteil (vgl. für eine eingehende Erläuterung Abschnitt 5.7). Der elterliche Stil der Vermittlung wirkt demnach - wie Darling und Steinberg (1993) postulieren - direkt oder vermittelt über die «Offenheit für elterliche Einflussnahmen» («the child's openness to parental socialization», S. 493) moderierend auf den Einfluss der Botschaft ein, die diese auf das Kind entfaltet. Wie Skinner und Kolleginnen (2009) schreiben:

The key notion is that interacting with parents who support children's fundamental psychological needs serves an energetic function. Children are motivated to constructively engage with parents, to cooperate with the parental agenda, and to internalize the behaviors and values promulgated by parents. In other words, they are ready to be socialized. In contrast, children who interact with parents who are hostile, chaotic, and coercive become disaffected from parent - child interactions, and can be sullen, submissive, oppositional, or apathetic. In other words, they resist socialization. (Skinner, E. A. et al., 2009, S. 190)

Ebenso von großer Bedeutung für die vorliegende Studie ist der Umstand, dass elterliche Erwartungen an die Kompetenz des Kindes sowie elterliche Aspirationen mit Blick auf Leistungsmaße der Kinder die bei weitem effektstärksten elterlichen Konstrukte bezüglich der schulbezogenen Unterstützung sind. Während interpersonelle behaviorale Erwartungen die Überzeugungen und Einschätzungen der Eltern davon bezeichnen, welches Kompetenzniveau ihr Kind realistischerweise erreichen wird, bezeichnen Aspirationen elterliche Hoffnungen bezüglich des Kompetenzniveaus, welches das Kind erreichen soll (vgl. Goldenberg et al., 2001, S. 548). Wie in Abschnitt 4.2 im Rahmen der Vorstellung des «Modells motivations- und leistungsbezogener Sozialisation im Elternhaus» (vgl. Abbildung 4.1) noch näher erörtert wird, reflektieren beide interpersonellen Kognitionen einerseits vergangene Erfahrungen der Eltern mit dem Kind, andererseits aber auch ihre impliziten Theorien (z. B. über die Veränderlichkeit von intellektuellen Fähigkeiten), ihre Stereotype (z. B. geschlechtsspezifische 
Begabungen) und - wie Hoorens (2012, S. 144) hervorstreicht - auch die Projektionen ihres eigenen Verhaltens (z. B. zeigt sich, dass Individuen ihre eigenen sozialen Werte wie Individualismus, Altruismus oder Wettbewerbsorientierung in geteilten Situationen auch von ihrem Gegenüber erwarten). Dass Erwartungen die subjektive Realität formen, zeigt sich etwa im «Placebo-Effekt» (treatment belief effect) oder bezogen auf interpersonale Erwartungen im «PygmalionEffekt» (self-fulfilling prophecies) bzw. im sog. demand effect in experimentellen Untersuchungssettings, in denen Menschen (und Tiere) sich offenbar konform zur Erwartung des signifikanten Gegenübers verhalten (Hoorens, 2012). Psychologisch erklärt wird dieser Umstand mit dem Zusammenspiel zwischen dem erwartungsgeleiteten Verhalten der signifikanten Anderen - hier der Eltern - auf der einen Seite und dem entsprechend den wahrgenommenen demand characteristics angepassten Verhaltensweisen der Rezipienten - hier der Kinder - auf der anderen Seite (vgl. Bandura, 1992; Bandura, 1997, S. 86-106). Bezogen auf die Effekte der breit untersuchten Erwartungen von Lehrkräften auf das Lern- und Leistungsverhalten von Schülerinnen und Schülern halten Wigfield et al. (2006) in ihrer Forschungsübersicht fest:

These person-specific expectations may be one of the most direct social influences on students' feelings of competence in classrooms. The research indicates that teacherexpectancy effects are mediated by the ways in which teachers interact with the students for whom they have high versus low expectations [...]. Whether the effects are positive or negative depends on the exact nature of these interactions. For example, a teacher can respond to low expectation by providing the kinds of help and structure that increase the student's sense of competence and ability to master the material being presented. Alternatively, the teacher can respond in ways that communicate low expectations and little hope that the student will be able to master the material. In the latter case, the students' own sense of competence should decrease and the student should disengage from the classroom's learning agenda as much as is possible. [...] What is critical is how these perceptions translate into the teachers' actual behavioral interactions with each of the students in the class. (Wigfield et al., 2006, S. 976)

Dass das beschriebene Zusammenspiel zwischen den interpersonellen Erwartungen und den intrapersonellen Kompetenzüberzeugungen gleichermaßen auch in der Familie stattfindet, konnten Buff et al. (2011) im Rahmen der TRANSITIONStudie bei 459 Kindern und ihren Eltern längsschnittlich (1. Klasse, 3. Klasse und 6. Klasse) belegen. Es zeigte sich, dass sich die elterlichen kindbezogenen Erwartungen (bzw. «kindbezogenen Kompetenzüberzeugungen») stark auf Leistungsinformationen der Schule in Form von Noten, aber auch auf Geschlechtsstereotype («Jungen sind besser in Mathematik als Mädchen») sowie - bei Kontrolle der Leistungen - auf die Kompetenzüberzeugungen der Kinder selber abstützten, 
sich nicht nur über alle Messzeitpunkte als relevante Prädiktoren der Kompetenzüberzeugungen der Kinder erwiesen, sondern mit diesen zusammen auch jeweils die nachfolgenden Leistungen beeinflussten. Wie sich die elterlichen Erwartungen, Aspirationen und Ziele in «into [...] actual behavioral interactions» mit dem Kind «übersetzen» (Wigfield et al., 2006, S. 976), wie Eltern dies konkret sprachlich tun und welche kompetenzbezogenen Erwartungen und Ziele sie Kindern namentlich in der zugespitzten Leistungssituation eines unklaren Übertrittsentscheids nahebringen möchten, ist kaum erforscht und wird im Zentrum der vorliegenden Untersuchung stehen.

Bevor in Kapitel 4 der Frage nachgegangen wird, inwiefern die soziale Herkunft der Eltern deren schulbezogene Orientierungen und Überzeugungen sowie deren Motivierungshandeln prädiktiert und inwiefern sich für das Kind daraus allenfalls Benachteiligungen für seine Bildungskarriere und seine gesellschaftliche Teilhabe ergeben, widmet sich das folgende Kapitel 3 dem Kontext vor dessen Hintergrund sich die hier interessierende familiale Kommunikation abspielt: schulischen Übertritten, deren Organisation sowie allfälligen Benachteiligungen, die sich daraus für Kinder und Eltern allenfalls ergeben.

Open Access Dieses Kapitel wird unter der Creative Commons Namensnennung 4.0 International Lizenz (http://creativecommons.org/licenses/by/4.0/deed.de) veröffentlicht, welche die Nutzung, Vervielfältigung, Bearbeitung, Verbreitung und Wiedergabe in jeglichem Medium und Format erlaubt, sofern Sie den/die ursprünglichen Autor(en) und die Quelle ordnungsgemäß nennen, einen Link zur Creative Commons Lizenz beifügen und angeben, ob Änderungen vorgenommen wurden.

Die in diesem Kapitel enthaltenen Bilder und sonstiges Drittmaterial unterliegen ebenfalls der genannten Creative Commons Lizenz, sofern sich aus der Abbildungslegende nichts anderes ergibt. Sofern das betreffende Material nicht unter der genannten Creative Commons Lizenz steht und die betreffende Handlung nicht nach gesetzlichen Vorschriften erlaubt ist, ist für die oben aufgeführten Weiterverwendungen des Materials die Einwilligung des jeweiligen Rechteinhabers einzuholen.

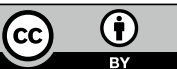

